\title{
Constructing stage-structured matrix population models from life tables: Comparison of methods
}

\author{
Masami Fujiwara ${ }^{\text {Corresp., }}{ }^{1}$, Jasmin Diaz-Lopez ${ }^{1}$ \\ ${ }^{1}$ Department of Wildlife and Fisheries Sciences, Texas A\&M University, College Station, Texas, United States \\ Corresponding Author: Masami Fujiwara \\ Email address: fujiwara@tamu.edu
}

A matrix population model is a convenient tool for summarizing per capita survival and reproduction rates (collectively vital rates) of a population and can be used for calculating an asymptotic finite population growth rate $(\lambda)$ and generation time. These two pieces of information can be used for determining the status of a threatened species. The use of stage-structured population models has increased in recent years, and the vital rates in such models are often estimated using a life table analysis. However, potential bias introduced when converting age-structured vital rates estimated from a life table into parameters for a stage-structured population model has not been assessed comprehensively. The objective of this study was to investigate the performance of methods for such conversions using simulated life histories of organisms. The underlying models incorporate various types of life history and true population growth rates of varying levels. The performance was measured by comparing differences in $\lambda$ and the generation time calculated using the Euler-Lotka equation, age-structured population matrices, and several stage-structured population matrices that were obtained by applying different conversion methods. The results show that the discretization of age introduces only small bias in $\lambda$ or generation time. Similarly, assuming a fixed age of maturation at the mean age of maturation does not introduce much bias. However, aggregating age-specific survival rates into a stage-specific survival rate and estimating a stage-transition rate can introduce substantial bias depending on the organism's life history type and the true values of $\lambda$. In order to aggregate survival rates, the use of the weighted arithmetic mean was the most robust method for estimating $\lambda$. Here, the weights are given by survivorship curve after discounting with $\lambda$. To estimate a stage-transition rate, matching the proportion of individuals transitioning, with $\lambda$ used for discounting the rate, was the best approach. However, stage-structured models performed poorly in estimating generation time, regardless of the methods used for constructing the models. Based on the results, we recommend using an age-structured matrix population model or the Euler-Lotka equation for calculating $\lambda$ and generation time when life table data are available. Then, these age- 
structured vital rates can be converted into a stage-structured model for further analyses. 
2

3 Constructing stage-structured matrix population 4 models from life tables: Comparison of methods

6 Authors: Masami Fujiwara, Jasmin Diaz-Lopez

7

8 Affiliation: Department of Wildlife and Fisheries Sciences, Texas A\&M University U.S.A.

11

12 Corresponding Author: Masami Fujiwara, fujiwara@tamu.edu 


\section{Abstract}

15 A matrix population model is a convenient tool for summarizing per capita survival and reproduction rates (collectively vital rates) of a population and can be used for calculating an asymptotic finite population growth rate $(\lambda)$ and generation time. These two pieces of information can be used for determining the status of a threatened species. The use of stagestructured population models has increased in recent years, and the vital rates in such models are often estimated using a life table analysis. However, potential bias introduced when converting age-structured vital rates estimated from a life table into parameters for a stage-structured population model has not been assessed comprehensively. The objective of this study was to investigate the performance of methods for such conversions using simulated life histories of organisms. The underlying models incorporate various types of life history and true population growth rates of varying levels. The performance was measured by comparing differences in $\lambda$ and the generation time calculated using the Euler-Lotka equation, age-structured population matrices, and several stage-structured population matrices that were obtained by applying different conversion methods. The results show that the discretization of age introduces only small bias in $\lambda$ or generation time. Similarly, assuming a fixed age of maturation at the mean age of maturation does not introduce much bias. However, aggregating age-specific survival rates into a stage-specific survival rate and estimating a stage-transition rate can introduce substantial bias depending on the organism's life history type and the true values of $\lambda$. In order to aggregate survival rates, the use of the weighted arithmetic mean was the most robust method for estimating $\lambda$. Here, the weights are given by survivorship curve after discounting with $\lambda$. To estimate a stage-transition rate, matching the proportion of individuals transitioning, with $\lambda$ used for discounting the rate, was the best approach. However, stage-structured models performed poorly in estimating generation time, regardless of the methods used for constructing the models. Based on the results, we recommend using an age-structured matrix population model or the Euler-Lotka equation for calculating $\lambda$ and generation time when life table data are available. Then, these age-structured vital rates can be converted into a stage-structured model for further 41 analyses. 


\section{Introduction}

43 A matrix population model is a convenient tool for summarizing per capita survival and reproduction rates (collectively vital rates) of a population, and is used for calculating an asymptotic finite population growth rate (commonly denoted by $\lambda$ ) and generation time. These two pieces of information can be used for determining the status of a threatened species (IUCN 2012). Matrix population models are broadly categorized into age-structured (Leslie matrix; Leslie 1945) and stage-structured (Lefkovitch matrix; Lefkovitch 1965) models. Age-structured matrix models group individuals based on age whereas stage-structured matrix models group individuals based on other properties such as developmental stage and size. Stage-structured matrix models are often favored when a property of individuals besides age is a better indicator of survival and reproduction (e.g. Caswell 2001; Cochran \& Ellner 1992). Although both types of models are common, the use of stage-structured population models has increased in recent years. Moreover, in many studies, vital rates in stage-structured population models may be estimated from age-structured vital rates through the grouping of age-classes together to form a stage (e.g. Brault \& Caswell 1993; Caswell et al. 1998; Crouse et al. 1987; Crowder et al. 1994). Typically a life table analysis is one of the main methods for obtaining age-specific vital rates.

life table data, survivorship (the proportion of age 0 that is alive at age $x$ ) is estimated, and then age-specific survival rates (the proportion of individuals that survive from age $x$ to $x+1$ ) are estimated. Age-specific survival rates along with age-specific fecundity rates can be entered into an age-structured matrix population model almost directly (see the Age-Structured Matrix Population Models section), and $\lambda$ and generation time can be calculated from the matrix. Matrix population models are also used for sensitivity and elasticity analyses (Caswell 1978; de Kroon et al. 2000). The inclusion of a large number of age-classes for long-lived organisms can make the interpretation of the sensitivity and elasticity analyses complicated because individuals in multiple age classes are often practically identical but separated in an age-structured model. rates into stage-specific vital rates, and to use stage-structured population matrices for calculating $\lambda$ and generation time. 
In order to convert age-specific into stage-specific vital rates, the former vital rates need

72 73 74 75 76

to be aggregated for the various stages. Furthermore, a transition rate from one stage to another needs to be estimated. Several approaches, or conversion methods, to aggregate survival rates for calculating a transition rate exist. However, the performance of the conversion methods has not been investigated comprehensively. Intuitively, the performance should depend on how survivorship and reproductive schedule change with age (life history) and whether a population is growing or declining in its abundance. Therefore, it is critically important to investigate the performance of the conversion methods for different life history and population growth scenarios.

The objective of this study is to investigate the performance of the conversion methods to estimate vital rates for stage-structured matrix population models from life tables. The performance is measured by comparing $\lambda$ 's and generation time calculated with the Euler-Lotka equation (see Kot 2001), age-structured population matrices, and several stage-structured population matrices obtained using different conversion methods. The asymptotic finite population growth rate $(\lambda)$ and the generation time calculated with the Euler-Lotka equation are considered the true values. Any discrepancies between the results obtained from the Euler-Lotka equation and those from an age-structured population matrix are considered to be due to bias introduced by discretizing age. In contrast, discrepancies between results from an age structured population matrix and those from stage-structured population matrices are considered to be due to bias introduced by conversion methods.

The comparisons were carried out with the life table data of organisms with different life history types. A wide range of life history types was incorporated into the analysis by artificially creating them using a competing risk model (Siler 1979) and three types of fecundity functions. However, the analysis focuses on those with a prolonged duration in a stage of interest. When the duration is short, stage structure is often embedded within age structure, and it is not necessary to convert age-structured vital rates into stage-structured rates. The comparisons were also repeated with life table data from populations that are declining $(\lambda<1)$, maintaining the same population density $(\lambda=1)$, or increasing $(\lambda>1)$ in population density in order to determine whether a stagestructured population matrix can be used for determining a population growth rate correctly under different population growth conditions. 
This paper is structured as follows. First, the life history types of organisms considered

101

102

103

104

\section{Models and Methods}

\section{Life History Types}

107 The types of life histories considered are broadly categorized into two groups: one with a short models to be described are listed in Table 1.

\section{Early Maturation Types}

120 For the early maturation types, two types of survivorship and three types of fecundity schedules

121 were incorporated. For the first type of survivorship, individuals experience a constant risk of

122 mortality. This leads to an exponentially declining survivorship curve $(l(x))$ with age: where $\alpha_{1}$ is the constant risk (i.e. hazard rate). For the second type of survivorship, individuals 


$$
l(x)=\mathrm{e}^{\frac{\alpha_{2}}{\beta_{2}}\left(1-e^{\beta_{2} x}\right)},
$$

127 where $\alpha_{2}$ and $\beta_{2}$ are the parameters for the increasing risk. Under this model, the hazard

128 function is given by $h(x)=\alpha_{2} e^{\beta_{2} x}$ (Siler 1979). This type of risk may be due to aging. These two 129 types of survivorship curves are shown in Figure 1A.

130 Figure 1B shows the three types of fecundity schedules. The first type assumes the

131 fecundity $b(x)$ is proportional to the cube of the body length of the individuals, which in turn

132 increases according to von Bertalanffy growth equation:

$$
b(x)=R_{1}\left[L_{\infty}\left(1-e^{-\kappa x}\right)\right]^{3},
$$

134 where $L_{\infty}$ and $\kappa$ are the parameters in the growth model (von Bertalanffy 1968). This assumes

135 136 reproduction is approximately proportional to the mass of individuals. The second type assumes a constant fecundity with age

$$
b(x)=R_{2} \text {. }
$$

Finally, the third type assumes exponentially declining fecundity:

$$
b(x)=R_{3} e^{-\gamma x}
$$

where $\gamma$ is the parameter determining how fast fecundity declines with age. Parameter $R_{m}$ (where $m=1,2$, or 3 ) are constants with different units depending on the type of fecundity, and these constants were used for adjusting $\lambda$ 's in this study (see Analytical Procedure). For all of the early maturation types in this study, individuals are assumed to mature at age 1.5 (i.e. $\left.x_{1}=x_{2}=1.5\right)$.

\section{Delayed Maturation Types}

For the delayed maturation types, two kinds of survivorship curves are incorporated into a juvenile stage. One incorporates a constant risk of mortality (Eq. 1), which results in exponentially declining survivorship (dashed curve in Figure 1C). The other assumes an exponentially declining risk of mortality in addition to a constant risk: 


$$
l(x)=\mathrm{e}^{-\alpha_{1} x} e^{\left[-\frac{\alpha_{3}}{\beta_{3}}\left(1-e^{-\beta_{3} x}\right)\right]},
$$

151 where $\alpha_{3}$ and $\beta_{3}$ are the parameters for the declining risk. In this model, the exponentially

152 declining hazard is given by $h(x)=\alpha_{3} e^{-\beta_{3} x}$ (Siler 1979). This causes initial fast descent of

153 survivorship (solid curve in Figure 1C). This type of survivorship can be observed when

154 organisms grow out of a mortality risk such as predation as they age. Once individuals mature,

155 they reach a constant risk of mortality (Eq. 1) and constant fecundity (Eq. 4.) under the delayed

156 maturation types.

In addition, three types of maturation schedule are incorporated. The first type assumes a fixed age of maturation at age 10.5 years $\left(x_{1}=x_{2}=10.5\right)$. The second type assumes a constant rate of maturation, and the third type assumes an exponentially increasing rate of maturation with age. In the latter two cases, maturation begins at age 8.5 years $\left(x_{1}=8.5\right)$, and the parameters are

161 chosen so that the mean age of maturity is approximately 10.5 years $\left(x_{2}=10.5\right)$; however, the mean age varies slightly depending on the level of mortality rate during the maturation period.

163 Under the latter two types of maturation schedules, the densities of individuals in immature (all

164 stages prior to maturation) and adult stages are described conveniently with a system of ordinary 165

166 differential equations (ODEs):

$$
\begin{aligned}
& \frac{d n_{1}(x)}{d x}=-m(x) n_{1}(x)-h(x) n_{1}(x), \\
& \frac{d n_{2}(x)}{d x}=m(x) n_{1}(x)-a n_{2}(x)
\end{aligned},
$$

167 where $n_{1}(x)$ and $n_{2}(x)$ are the densities of immature individuals and adults at age $x$,

168 respectively, $m(x)$ is a per-capita instantaneous maturation rate at age $x$, and $h(x)$ is the age-

169 dependent hazard function of mortality. For the second type of maturation schedule, $m(x)$ is set

170 at $m_{1}=0.5$ for $x \geq x_{1}$ and 0 otherwise. For the third type, $m(x)=m_{1} e^{m_{2}\left(x-x_{1}\right)}$ for $x \geq x_{1}$, and

$171 m(x)=0$ otherwise. The proportion of individuals that are mature at a given age for the three

172 types of maturation schedule is shown in Figure 1D. When the system of ODEs is solved with 
173 the initial condition $\left[n_{1}(0) \quad n_{2}(0)\right]=\left[\begin{array}{ll}1 & 0\end{array}\right]$ using a numerical ODE solver, the survivorship is

174 given by $l(x)=n_{1}(x)+n_{2}(x)$. Note equation 7 does not include reproduction, and the initial

175 condition is 1 in the immature stage; therefore, $l(x) \leq 1$ for all $x \geq 0$.

\section{Conversion Methods}

177 In order to convert age-structured vital rates into stage-structured vital rates, three types of vital

178 rates need to be calculated: a stage-specific survival rate for stage $i\left(S_{i}\right)$, a transition rate from

179 stage $i$ to stage $j$ conditional on their survival $\left(P_{j, i}\right)$, and a fertility rate for stage $i\left(F_{i}\right)$. The

180 methods for converting these vital rates are described in the following sections.

\section{Stage-Specific Survival Rate}

182 All the methods for calculating a stage-specific survival rate from age-specific survival rates

183 investigated in this study assume that the beginning and ending ages of a stage are given by the

184 mean ages of transition into and from the stage, respectively, and that these ages are known. The

185 former assumption can potentially produce bias when calculating $\lambda$ and generation time because

186 stage transitions often do not occur at a fixed age (i.e. individuals gradually transition from one

187 stage to another over a range of ages). Therefore, the performance of conversion methods was

188 also investigated when the age of stage-transitions was not fixed (Eq. 7). It is also possible that

189 stage-transitions are completely age-independent (e.g., as in some plant species). This situation is

190 beyond the scope of the current analysis, but it will be briefly discussed in the Discussion section.

An age-specific survival rate from age $x$ to $x+1$ is given by

$$
s_{x}=\frac{l(x+1)}{l(x)} .
$$

194 Hereafter, lower case $s$ is used for denoting an age-specific survival rate whereas the upper case

$195 S$ is used for denoting a stage-specific survival rate. Suppose the stage begins at age $x^{(i)}$ and

196 ends at $x^{(j)}-1$ (i.e. age $x^{(j)}$ is the next stage), then the survival rate of individuals in the stage is

197 given by the mean survival rate over age classes within the stage. Note an index in the

198 superscript within parentheses denote a stage. Here, nine different ways of calculating the mean 
199 are compared. First, the mean is given by geometric mean, arithmetic mean, or harmonic mean

200 (Table 2). These are three common methods for obtaining a stage-specific survival rate. However,

201 the number of individuals in age classes are different, and it is more appropriate to put weight

202 based on the proportion of individuals in a given age class, which is given by a survivorship

203 curve. This leads to a weighted geometric mean, weighted arithmetic mean, and weighted

204 harmonic mean (Table 2). Although weighting with survivorship is fine when a population is

205 neither growing nor declining (i.e. $\lambda=1$ ), when a population is growing (or declining), there are

206 more (or less) individuals in younger age classes than predicted by a survivorship curve.

207 Therefore, it is necessary to discount the weight using $\lambda$. This leads to weighted means where the

208 weight is given by both survivorship and $\lambda$ (Table 2). Hereafter, the latter weight is termed "a

209 discounted weight."

210 Conditional Transition Rate

211 Transition rate from stage $i$ to stage $j$ conditional on their survival $\left(p_{j, i}\right)$ is calculated in three

212 ways (Table 3 ). The first method (T1) matches the expected duration in the stage assuming

213 exponentially declining time to transition into the following stage. The second method (T2)

214 matches the expected proportion of individuals making the transition into the following stage

215 assuming the survivorship curve gives the distribution of individuals among age-classes. The

216 third method (T3) is the same as the second method except that the distribution is discounted by

217 a population growth rate. These methods are described in more detail in Caswell (2001).

\section{Fertility Rate}

219 Fertility rates in matrix population models are different from fecundity in a life table. A fertility

220 rate gives the per capita rate of contribution from one stage to the next over one year so that

221 adults will have to survive to reproduce, and/or offspring must survive to appear in the next stage.

222 On the other hand, fecundity is the number of offspring produced. The latter does not include any

223 survival of adult or offspring. Consequently, there are two steps in converting life table data into

224 a stage-structured fertility rate: calculating age-specific fertility rates and converting them into a

225 stage-specific fertility rate. There are many approaches for the first step depending on the

226 reproductive schedules of organisms within a year (see Caswell 2001) or among years (e.g.

227 Crowder et al. 1994). Evaluating them is beyond the scope of this study. Here, reproduction was 
228 assumed to occur at any time of year (i.e. a birth flow model), and an age-specific fertility rate ( $229 f_{x}$ ) was estimated as follows:

230

$$
f_{x}=l(0.5) \int_{z=x-0.5}^{x+0.5} b(z) \frac{l(z)}{l(x-0.5)} d z
$$

231 where $b(z)$ is the instantaneous per-capita fecundity rate at age $x$. In the equation,

$232 l(z) / l(x-0.5)$ gives the survival rate of adults from age $x-0.5$ to age $z$ (where $z>x-0.5)$,

233 and the integral calculates the total number of offspring produced between ages $x-0.5$ and

$234 x+0.5$ per adult that was alive at age $x-0.5$. Then, all births are attributed to the half way

235 point (i.e. age $x$ ) so that offspring will have to survive half a year on average to appear in the

236 first stage; this survival rate is given by $l(0.5)$, which is the survival rate from age 0 to 0.5 .

237

238

239

240

Once age-specific fertility rates are obtained, a stage-specific fertility rate $F_{i}$ is calculated in three different ways. First, an arithmetic mean is taken with equal weights on all age classes,

$$
F_{i}=\frac{1}{x_{3}-x_{2}} \sum_{x=x_{2}+0.5}^{x_{3}-0.5} f_{x}
$$

The second approach is to take a weighted arithmetic mean,

$$
F_{i}=\frac{1}{\sum_{x=x_{2}+0.5}^{x_{3}-0.5} \omega_{1, x}} \sum_{x=x_{2}+0.5}^{x_{3}-0.5} \omega_{1, x} f_{x}
$$

242 where $\omega_{1, x}=l(x)$. The third method is to use a discounted weight by replacing $\omega_{1, x}$ in equation

24311 with $\omega_{2, x}=\frac{1}{\lambda^{x-x_{0}}} l(x)$. In all calculations, the same weight (or no weight) is used for

244 calculating a stage-specific survival rate and a stage-specific fertility rate.

\section{Asymptotic Population Growth Rate and Generation Time}

246 An asymptotic population growth rate (a finite per capita population growth rate $\lambda$ ) and

247 generation time are calculated using the Euler-Lotka equation, age-structured (Leslie) matrices, 
248 and stage-structured (Lefkovitch) matrices. These models and the calculations of $\lambda$ and

249 generation time are described in this section.

250 Euler-Lotka Equation

251 The Euler-Lotka equation (Kot 2001) is given by

252

$$
1=\int_{0}^{x_{3}} \lambda^{-x} b(x) l(x) d x
$$

253 The fecundity $b(x)$ and survivorship $l(x)$ are defined for a specific life history type as

254 described in the previous section (Figure 1). Provided $R_{m}$ is fixed, the only unknown is $\lambda$, which 255 can be found numerically by searching the value that satisfies equation (12) using a root-finding 256 algorithm.

257 Generation time in this study is defined as the mean age of parents where the mean is 258 calculated over all offspring born at a given time. More specifically, generation time $G_{1}$ is given 259 by

$$
G_{1}=\int_{0}^{x_{3}} x \lambda^{-x} b(x) l(x) d x
$$

261 (Keyfitz \& Caswell 2005). In the case where individuals mature over a range of ages, some 262 individuals do not reproduce in certain age ranges. Therefore, generation time $G_{2}$ is instead 263 given by

$$
G_{2}=\int_{0}^{x_{3}} x \lambda^{-x} b(x) q(x) l(x) d x,
$$

265 where $q(x)$ is the proportion of individuals that are mature at age $x$.

266 Age-Structured Matrix Population Models (Leslie Matrix)

267 An age-structured population matrix is given as 


$$
\mathbf{A}=\left[\begin{array}{ccccc}
0 & f_{2} & \ldots & f_{x_{3}-1.5} & f_{x_{3}-0.5} \\
s_{0.5} & 0 & \ldots & 0 & 0 \\
0 & s_{1.5} & \ldots & 0 & 0 \\
\cdots & \cdots & \ldots & \ldots & \cdots \\
0 & 0 & \ldots & s_{x_{3}-2} & 0
\end{array}\right]
$$

269 In this study, the first age class is assumed to begin at age 0.5, and subsequent age class is

270 incremented by one year. It should be noted that survival rate $s_{x}$ gives the proportion of

271 individuals that survive from time $x$ to $x+1$ and fertility $f_{x}$ gives the mean number of offspring

272 of age 0.5 produced by a parent alive at age $x-0.5$ by reproducing between age $x-0.5$ and

$273 x+0.5$ (see equation 9). It is assumed that the reproduction begins at the mean age of maturation $274 x_{2}$.

275 In the case where individuals mature over a range of age, the population matrix is given

276 instead as

$$
\mathbf{A}=\left[\begin{array}{ccccc}
0 & q_{2} f_{2} & \ldots & q_{x_{3}-1.5} f_{x_{3}-1.5} & q_{x_{3}-0.5} f_{x_{3}-0.5} \\
s_{0.5} & 0 & \ldots & 0 & 0 \\
0 & s_{1.5} & \ldots & 0 & 0 \\
\cdots & \cdots & \ldots & \ldots & \cdots \\
0 & 0 & \ldots & s_{x_{3}-2} & 0
\end{array}\right] .
$$
eigenvalue. Furthermore, generation time is given by

$$
G_{3}=\frac{\lambda \mathbf{v w}}{\mathbf{v F w}}
$$

281 where $\mathbf{v}$ and $\mathbf{w}$ are the left and right eigenvectors (row and column vectors), respectively, of the

282 population matrix, and $\mathbf{F}$ is a modified population matrix only consisting of fertility terms

283 (Bienvenu \& Legendre 2015).

\section{Stage-Structured Matrix Population Models (Lefkovitch Matrix)}

285 Stage-structured matrix population models are given in two different forms in this study. For 286 early maturation types, a two-stage population matrix is used 


$$
\mathbf{A}=\left[\begin{array}{cc}
0 & F_{2} \\
S_{1} & S_{2}
\end{array}\right]
$$

288

where the duration of stage 1 is from age $x_{0}=0.5$ to age $x_{1}=x_{2}=1.5$ and the duration of stage 2 is from age 1.5 to age $x_{3}=40.5$.

For organisms with delayed maturation, three-stage matrix population models are used

$$
\mathbf{A}=\left[\begin{array}{ccc}
0 & 0 & F_{3} \\
S_{1} & S_{2}\left(1-P_{3,2}\right) & 0 \\
0 & S_{2} P_{3,2} & S_{3}
\end{array}\right]
$$

292 The duration in stages 1,2 , and 3 are assumed to be from age $x_{0}=0.5$ to 1.5 , from age 1.5 to

$293 x_{1}=x_{2}=10.5$, and from age $x_{2}=10.5$ to $x_{3}=40.5$. Using the stage-structured matrix, $\lambda$ and

294 generation time can be calculated in the same way as with the age-structured matrices.

295

296

297

298

299

300

301

302

303

304

305

306

307

308

309

310

\section{Analytical Procedure}

Thirty-six different scenarios of life history strategies and a population growth rate were investigated in this study. For the early maturation type, there were six different life history strategies (two types of survivorships and three types of fecundity schedules; Figure 1A and 1B). With each of the six life history strategies, the true finite asymptotic population growth rate $(\lambda)$ was adjusted to $0.900,1.000$, and 1.100 by adjusting coefficient $R_{m}$ in the fecundity function (Eq. 3,4 , and 5). This adjustment was done with the Euler-Lotka equation by searching the value $R_{m}$ that gives the corresponding value of $\lambda$. Consequently, there were 18 early maturation type scenarios to investigate. For each scenario, one age-structured population model and nine twostage population models were constructed; the nine models differ in the ways that adult survival rate and a fertility rate were calculated (see Table 2 ). Then, with each model, $\lambda$ and generation time were calculated.

Similarly, for the delayed maturation type, there were six different life history strategies (two types of survivorships and three types of maturation schedules; Figure 1C and 1D). For each type, finite asymptotic population growth rate $(\lambda)$ was also adjusted to $0.900,1.000$, and 1.100. Then, two age-structured population models (Eq. 15 and 16) and nine three-stage 
311 population models were constructed; these nine models differ in the ways that a juvenile survival

312 rate was aggregated and a conditional transition rate was obtained (see Table 2). Then, with each

313 model, $\lambda$ and generation time were calculated.

314 In order to obtain the true generation time, equation 13 was used when organisms had a

315 fixed age of maturation, while equation 14 was used when organisms matured over a range of

316 age. All of the calculations were done with MATLAB (MATLAB 2012). For solving a system of

317 ODE's, a built-in ODE solver "ode45" was used in most cases. When a maturation rate was

318 exponentially increasing with age, there was a numerical problem with the function (equations

319 became stiff). Therefore, "ode15s" was used, instead. For the methods that require $\lambda$ in

320 aggregating a survival rate, a transition rate, and/or a fertility rate, the true $\lambda$ was used.

321 In actual analyses conducted with real data, estimating $\lambda$ is one of the main purposes of

322 constructing a population matrix. In other words, it is not known a priori. To overcome this issue,

323 Caswell (2001) describes an iterative method. In this method, an initial $\lambda$ is arbitrarily selected

324 and used for obtaining factors needed to calculate the conditional transition rate. Then, $\lambda$ is

325 estimated from the obtained population matrix. Subsequently, the new $\lambda$ is used to obtain a new

326 population matrix and $\lambda$ is calculated again. This process is repeated until $\lambda$ converges. For

327 example, this method was used for developing a stage-structured population matrix of

328 loggerhead sea turtles (Crowder et al. 1994). In the current study, this procedure is applied to

329 delayed maturation life history strategy. Discounted-weight arithmetic mean was used for

330 aggregating juvenile and adult survival rates and a transition rate was obtained by matching

331 proportion transitioning also using $\lambda$ as a discounting factor.

\section{Results}

\section{Finite Asymptotic Population Growth Rate}

334 Figures 2-7 show estimated finite per capita population growth rates $(\lambda)$. In each figure, each

335 panel shows the estimated $\lambda$ of the same life history type calculated using different methods.

336 These methods are listed in Table 4. The first bar (black) is the true value, and the rest of the bars

337 are obtained with age- or stage-structured population matrices.

338 For the early maturation types (Figures 2-4), one age-structured model and nine stage-

339 structured models that differ in the methods for calculating an adult survival rate were used. The 
340 three figures are different in the true $\lambda$ 's. For all life history types and all true $\lambda$ 's (Figures 2-4),

341 the Euler-Lotka equation (model a) and age-structured model (model c) show the similar values

342 (black and gray bars in Figures 2-4). This suggests there is no bias introduced by discretizing age.

343

344 When adults experience a constant risk of mortality (Eq. 1) and constant fecundity (Eq. 4),

345 all conversion methods performed well (within $2 \%$ of the true value; see panel B of Figures 2-4).

346 In all other cases, some of the methods performed poorly (see panel A and panels C-F of Figures 347 2-4).

Comparing the use of the geometric mean, arithmetic mean, and harmonic mean of adult survival rate (Models d-f in panels A-C of Figures 2-4), all gave the similar results for adults experiencing a constant risk. However, for adults experiencing an exponentially increasing risk (panels D-F), the three means (Models d-f) gave different results. In particular, the harmonic mean (Model f) grossly underestimated $\lambda$, as did the geometric mean (Model d) although to a lesser extent. Among the three, the arithmetic mean (Model e) performed better, but it still underestimated $\lambda$.

Weighted means (Models g-i) improved the estimation of $\lambda$, compared with unweighted means (Models d-e). For example, when the weighted arithmetic mean was used, declining populations $(\lambda=0.9)$ were always identified as declining $(\lambda<1)$, and increasing populations $(\lambda=1.1)$ were always identified as increasing $(\lambda>1)$. The use of discounted weight (Models $\mathrm{j}-\mathrm{k})$ further improved the estimation of $\lambda$, especially, when $\lambda$ was not at 1.0 (Figures 3-4).

For the delayed maturation types, two age-structured models and nine stage-structured models were used (Figures 5-7). The two age-structured models differed in whether the age of maturation was assumed to be fixed at the mean age of maturity (Model b; Eq. 15) or the proportion of mature individuals at a given age is incorporated (Model c; Eq. 16). The nine stage-structured models differed in the method for calculating the conditional transition rate (Table 3) and in how juvenile survival was aggregated (Table 2). For the latter aggregation, arithmetic mean, weighted arithmetic mean, and discounted-weight arithmetic mean were used.

367 First, age-structured models gave $\lambda$ consistent with the true value (cf. Model a and Models b-c of all panels of Figures 5-7). This suggests discretization of age did not introduce bias in $\lambda$.

369 Furthermore, the incorporation of the proportion of mature individuals did not improve $\lambda$ (cf. 
370 Model $\mathrm{b}$ and $\mathrm{c}$ of all panels of Figures 5-7), suggesting the assumption of the fixed age of 371 maturation was appropriate.

372 For stage-structured models, bias introduced in the estimation of $\lambda$ was not as large as that 373 with the early maturation types. Among the three conditional transition rate estimation methods 374 (Table 3), the third method (T3) to match the proportion of individuals making the transition 375 after discounting with $\lambda$ (Models $\mathrm{o}, \mathrm{r}$, and $\mathrm{u}$ ) performed best, and the first method to match the 376 average duration (Models $\mathrm{m}, \mathrm{p}$, and s) performed the worst. The estimation was less sensitive to 377 how the juvenile survival rate was aggregated.

378 Generation Time

379 Generation time is shown in Figures 8-13. In all cases, age-structured models (Model b in 380 Figures 8-10 and Models $\mathrm{b}$ and $\mathrm{c}$ in Figures 11-13) gave a generation time that was consistent 381 with the true values (Model a). This suggests discretization of age does not introduce bias.

382 However, stage-structured models gave substantially different values for most scenarios. They 383 gave similar results to the true values only for populations experiencing constant hazard and 384 constant fecundity for early maturation types as long as the population was steady ( $\lambda=1$, panel B

385 of Figure 8 ) or growing ( $\lambda>1$, panel B of Figure 10). These results suggest generation time 386 obtained from stage-structured models are generally not accurate.

\section{Iterative Methods}

388 All of the iterative methods in this study converged very quickly. The estimated $\lambda$ value obtained using the iterative method is shown in Table 5. Overall, the iterative method performed well in estimating $\lambda$. It was accurate to the first digit of $\lambda$ at least. However, generation time was estimated almost equally poorly with that obtained assuming $\lambda$ was known a priori (i.e. Model $\mathrm{u}$ of all panels of Figures 11-13).

\section{Discussion}

394 Population growth rate $(\lambda)$ and generation time are two of the most important pieces of 395 information in determining the status of threatened species (see IUCN 2012). The former tells us 396 how quickly a population is expected to be declining or growing over time, and the latter tells us 397 an appropriate time-scale for a population. Accurate determination of these parameters is 398 essential. For example, the overestimation of generation time or underestimation of $\lambda$ will result 
399 in species being erroneously placed in categories of higher threat than they should be. In this

400 study, we have evaluated the performance of stage-structured population models in calculating $\lambda$

401 and generation time, when life table data are used to parameterize the models.

402 Overall, discretization of age does not introduce much bias in population growth rate $(\lambda)$

403 or generation time (comparing the first and second bars of all panels of Figures 2-13). Similarly,

404 assuming a fixed age of maturation at the mean age of maturity, does not introduce much bias

405 (comparing the second and third bars of all panels of Figures 5-7 and Figures 11-13). Although

406 only three types of maturation schedule have been investigated in this study, the latter conclusion

407 is expected to remain true as long as the age-range of maturation is not substantially wide. On the

408 other hand, the aggregations of age-specific vital rates into a stage-structured vital rate introduces 409 bias.

410 In order to aggregate survival rates for stage-structured population matrices, the use of

411 the discounted-weight arithmetic mean is the most robust method to estimate $\lambda$ (Figures 2-4).

412 However, the method requires the use of $\lambda$ to discount the weight, which defeats the purpose of

413 constructing a stage-structured population model to obtain $\lambda$. Therefore, the use of the weighted

414 arithmetic mean is a better option although it can still introduce some bias when the population is

415 either growing or declining. For the calculation of the conditional transition rate, the best

416 approach is to match the proportion transitioning, with $\lambda$ being used as a discounting factor;

417 however, the use of the method without the discounting factor also performs well (Figures 5-7).

418 The study also suggests that aggregating adult stage needs to be done more carefully than 419 aggregating juvenile stage (compare Figures 2-4 and Figures 5-7). Adults experience survival 420 and reproduction whereas juveniles experience survival and maturation. Because they experience 421 different population processes, it is not surprising to see the difference. However, the results may 422 also be because the adult stage is much longer than the juvenile stage in this study. For example, 423 some organisms exhibit a short-lived adult stage with delayed maturation. For such organisms, 424 the aggregation for the juvenile stage would need to be done more carefully than for the adult 425 stage.

426 One of the problems is the heterogeneity among individuals within a stage. In our model, 427 the heterogeneity exists because either the risk of mortality or the fecundity rate changes with 428 age. However, a matrix population model assumes that all individuals are the same within a stage. 
429 When both mortality risk and fecundity rate are constant over age, $\lambda$ and generation time are 430 estimated accurately with stage-structured population models (see second columns of Figure 2 431 and Figure 7). However, variation in mortality with age is commonly experienced. For example, 432 young individuals may grow out of a predation risk, and older individuals may experience 433 senescence. The former is common among any animal populations, and the latter is common 434 among long-lived mammals. Fecundity also changes with age. For example, it is often a function 435 of the size of adults, which increases with age, a common change seen in fish. One way to 436 overcome the issue of a non-constant mortality risk or non-constant fecundity may be to 437 aggregate smaller number of age classes into one stage by including a large number of stages in a 438 model. This could be done after fitting a competing risk model (Siler 1979) and a flexible 439 fecundity function to life table data and examining the age classes with a similar mortality risk 440 and fecundity.

Our results suggest estimating generation time is more problematic than estimating $\lambda$. For example, when fecundity was declining with age under the early maturation model (panel $\mathrm{C}$ in Figure 7), generation time was always overestimated with stage-structured models. This is because although in reality younger individuals contribute more to reproduction than older individuals do, stage-structured models assume homogeneity among adults. In other words, older adults contribute more in the models than the true population. Consequently, generation time is overestimated. When fecundity was declining with age (panel A in Figure 7), the opposite effect was observed. When survival rate was changing with age, it can lead to over- or underestimation of generation time, and it is difficult to predict. For these reasons, the construction of an age structured model or the use of the Euler-Lotka equation is recommended for calculating $\lambda$ and generation time. models to calculate generation time. Lebreton recommends the use of stage-structured models in which stages are embedded within age classes. This effectively increases the number of stages in a model. Such a population matrix can be constructed if one collects information on the stage of individuals in addition to age in order to construct a life table in which individuals are categorized into different age and stage classes. This approach is also useful when life history strategies are complex or when they do not exhibit age-dependent changes in vital rates. For such 
459 organisms, a simple life table alone may not be informative because each age class can include 460 multiple stages, but a stage-structured model embedded in age structure should provide accurate 461 estimations of both $\lambda$ and generation time. Salguero-Gomez \& Plotkin (2010) also discusses the 462 relationship between the number of stages and various statistics obtained from stage-structured 463 population matrices.

An additional problem is the change in stage/age distribution due to growing or declining population abundance. A population growth rate needs to be discounted when a survivorship curve is estimated from a life table. Discounting is required for the same reason that it is included in the Euler-Lotka equation. There are two types of life table, dynamic or static life table. A dynamic life table is also called a cohort life table because it is constructed using data obtained by following the same cohort over time/age. On the other hand, a static life table is obtained by examining the age distribution of samples collected at one sampling occasion. Throughout this paper, a dynamic life table has been assumed. When a static life table is collected instead, a survivorship curve needs to be discounted by a population growth rate to obtain the true survivorship curve. The discounting is necessary because individuals in different age classes in the data were born at different time and the population is growing or declining so that increasing or decreasing numbers of individuals are born from one year to the next. Interestingly, the age distribution obtained directly from a static life table gives the discounted weight used in this 477 study.

The iterative method allows the use of $\lambda$ in constructing a stage-structured population matrix without knowing $\lambda$ a priori. This method performed well in identifying whether $\lambda$ is greater or smaller than 1. In this sense, the iterative method is a viable option for constructing a stage-structured population model. However, when a life table is available, an age-structured matrix population model can be used for calculating $\lambda$ more accurately. Furthermore, an agestructured matrix allows accurate estimation of generation time, which can be substantially biased in stage-structured population models.

In this study, bias was introduced when constructing matrix population models using information from life table data. As an alternative to life table data, Cormack-Jolly-Seber (CJS) type capture-recapture data (Lebreton et al. 1992) can be obtained. With additional information 
489 recapture method can be used for estimating parameters in stage-structured matrix population

490 models directly (Fujiwara \& Caswell 2002; Nichols et al. 1992). Provided there is no

491 heterogeneity in a capture rate within a given stage, the method accounts for an underlying age-

492 distribution although an actual age distribution may not be observable. When age is not known,

493 the use of stage-structured population models is the only option. However, generation time

494 obtained from such models may not be accurate. Future investigations of the performance of

495 generation time calculations with stage-structured population models when vital rates are

496 estimated from individual capture-recapture data are needed.

\section{Conclusions}

498 When life table data are collected, we recommend fitting a competing risk model and a flexible

499 fecundity function to the data and estimating population growth rate $\lambda$ and generation time using 500 an age-structured population matrix or the Euler-Lotka equation. Calculating generation time

501 using stage-structured population models should be avoided. If a researcher is interested in

502 constructing stage-structured population models (e.g. for the purpose of sensitivity and elasticity 503 analyses), the conversion from age-structured vital rates to stage-structured vital rates should be

504 done by aggregating age classes with similar mortality risk and fecundity into the same stage.

505 When aggregating survival rates for constructing stage-structured population models,

506 discounted-weight arithmetic mean should be used. When calculating the conditional transition

507 rate, one should use the method for matching the proportion making transitions with $\lambda$ as a

508 discounting factor.

\section{References}

510 Bienvenu F, and Legendre S. 2015. A new approach to the generation time in matrix population models. American Naturalist 185:834-843. DOI: 10.1086/681104

512 Brault S, and Caswell H. 1993. Pod-specific demography of killer whales (Orcinus orca). Ecology 74:1444-1454. DOI: 10.2307/1940073

514 Caswell H. 1978. General formula for sensitivity of population-growth rate to changes in lifehistory parameters. Theoretical Population Biology 14:215-230. DOI: 10.1016/00405809(78)90025-4 
517 Caswell H. 2001. Matrix Population Models: Construction, Analysis, and Interpretation.

$518 \quad$ Sunderland, MA: Sinauer Associates, Inc.

519 Caswell H, Brault S, Read AJ, and Smith TD. 1998. Harbor porpoise and fisheries: An

520 uncertainty analysis of incidental mortality. Ecological Applications 8:1226-1238. DOI:

$521 \quad$ 10.1890/1051-0761(1998)008[1226:HPAFAU]2.0.CO;2

522 Cochran ME, and Ellner S. 1992. Simple methods for calculating age-based life-history

523 parameters for stage-structured populations. Ecological Monographs 62:345-364. DOI:

$524 \quad 10.2307 / 2937115$

525 Crouse DT, Crowder LB, and Caswell H. 1987. A stage-based population-model for Loggerhead 526 sea-turtles and implications for conservation. Ecology 68:1412-1423. DOI:

$527 \quad 10.2307 / 1939225$

528 Crowder LB, Crouse DT, Heppell SS, and Martin TH. 1994. Predicting the impact of turtle

529

530 excluder devices on Loggerhead sea-turtle populations. Ecological Applications 4:437445. DOI: $10.2307 / 1941948$

531

532

533

534

de Kroon H, van Groenendael J, and Ehrlen J. 2000. Elasticities: A review of methods and model limitations. Ecology 81:607-618. DOI: 10.1890/00129658(2000)081[0607:EAROMA]2.0.CO;2

Fujiwara M, and Caswell H. 2002. Estimating population projection matrices from multi-stage mark-recapture data. Ecology 83:3257-3265. DOI: 10.2307/3072076

IUCN. 2012. IUCN Red List Categories and Criteria: Version 3.1. Second edition. Gland, Switzerland and Cambridge, UK.

Keyfitz N, and Caswell H. 2005. Applied Mathematical Demography, $3^{r d}$ Ed. Statistics for Biology and Health. Springer, New York, U.S.A.

540 Kot M. 2001. Elements of Mathematical Ecology. Cambridge, U.K.: Cambridge University Press.

541 Lebreton JD. 2005. Age, stages, and the role of generation time in matrix models. Ecological 
543 Lebreton JD, Burnham KP, Clobert J, and Anderson DR. 1992. Modeling survival and testing 544 biological hypotheses using marked animals - a unified approach with case-studies.

545 Ecological Monographs 62:67-118. DOI: 10.2307/2937171

546 Lefkovitch LP. 1965. Study of population growth in organisms grouped by stages. Biometrics 21:1-18. DOI: $10.2307 / 2528348$

548 Leslie PH. 1945. On the use of matrices in certain population mathematics. Biometrika 33:183212. DOI: $10.2307 / 2332297$

MATLAB. 2012. Ver. 7. Natic, Masacchusetts, U.S.A.: The MathWorks, Inc.

551 Nichols JD, Sauer JR, Pollock KH, and Hestbeck JB. 1992. Estimating Transition-Probabilities 552 For Stage-Based Population Projection Matrices Using Capture Recapture Data. Ecology 73:306-312. 10.2307/1938741

554 555

556

557 558

559

560

561
Salguero-Gomez R, and Plotkin JB. 2010. Matrix Dimensions Bias Demographic Inferences: Implications for Comparative Plant Demography. American Naturalist 176:710-722. $10.1086 / 657044$

Siler W. 1979. A competing-risk model for animal mortality. Ecology 60:750-757. DOI: $10.2307 / 1936612$

von Bertalanffy L. 1968. General System Theory: Foundations, Development, Applications. New York: George Braziller, Inc. 


\section{Table 1}

563 Table 1. Parameters for twelve different life history types.

\begin{tabular}{|c|c|c|c|c|c|c|c|c|c|c|c|c|c|}
\hline \multicolumn{10}{|c|}{ Early Maturation Type } & \multirow[b]{2}{*}{$x_{0}$} & \multirow[b]{2}{*}{$x_{1}$} & \multirow[b]{2}{*}{$x_{2}$} & \multirow[b]{2}{*}{$x_{3}$} \\
\hline Model & $\alpha_{1}$ & $\alpha_{2}$ & $\beta_{2}$ & $L_{\infty}$ & $\kappa$ & & $R_{\lambda=0.9}^{-1}$ & $R_{\lambda=1.0}^{-1}$ & $R_{\lambda=1.1}^{-1}$ & & & & \\
\hline $\begin{array}{l}\text { Dimen } \\
\text { sion }\end{array}$ & $\tau^{-1}$ & $\tau^{-1}$ & $\tau^{-1}$ & $l$ & $\tau^{-1}$ & & $\mu^{-1} \tau l^{3}$ & $\mu^{-1} \tau$ & $\mu^{-1} \tau$ & $\tau$ & $\tau$ & $\tau$ & $\tau$ \\
\hline $\mathrm{CH}-\mathrm{IF}$ & .1535 & 0 & -- & 10 & .10 & & 6832 & 948 & 280 & 1.5 & 1.5 & 1.5 & 40.5 \\
\hline $\mathrm{CH}-\mathrm{CF}$ & .1535 & 0 & -- & 10 & -- & & 16371 & 5163 & 2768 & 1.5 & 1.5 & 1.5 & 40.5 \\
\hline CH-DF & .1535 & 0 & -- & 10 & .10 & & 6262 & 3134 & 1975 & 1.5 & 1.5 & 1.5 & 40.5 \\
\hline IH-IF & 0 & .002 & .205 & 10 & .10 & & 39459 & 5985 & 1435 & 1.5 & 1.5 & 1.5 & 40.5 \\
\hline IH-CF & 0 & .002 & .205 & 10 & -- & & 80276 & 18485 & 7244 & 1.5 & 1.5 & 1.5 & 40.5 \\
\hline IH-DF & 0 & .002 & .205 & 10 & .10 & & 22872 & 8100 & 4176 & 1.5 & 1.5 & 1.5 & 40.5 \\
\hline \multicolumn{14}{|c|}{ Delayed Maturation Type } \\
\hline Model & $\alpha_{1}$ & $\alpha_{3}$ & $\beta_{3}$ & $m_{1}$ & $m_{2}$ & $a$ & $R_{\lambda=0.9}^{-1}$ & $R_{\lambda=1.0}^{-1}$ & $R_{\lambda=1.1}^{-1}$ & $x_{0}$ & $x_{1}$ & $x_{2}$ & $x_{3}$ \\
\hline $\begin{array}{c}\text { Dimen } \\
\text { sion }\end{array}$ & $\tau^{-1}$ & $\tau^{-1}$ & $\tau^{-1}$ & $g \tau^{-1}$ & $\tau^{-1}$ & $\tau^{-1}$ & $\mu^{-1} \tau l^{3}$ & $\mu^{-1} \tau$ & $\mu^{-1} \tau$ & $\tau$ & $\tau$ & $\tau$ & $\tau$ \\
\hline DH-FM & .1000 & .400 & .300 & -- & -- & .20 & 3.000 & 0.497 & 0.124 & 1.5 & 10.5 & 10.5 & 40.5 \\
\hline CH-FM & .2193 & 0 & -- & -- & -- & .20 & 3.005 & 0.498 & 0.124 & 1.5 & 10.5 & 10.5 & 40.5 \\
\hline DH-CM & .1000 & .400 & .300 & .50 & 0 & .20 & 2.958 & 0.502 & 0.131 & 1.5 & 8.5 & 10.5 & 40.5 \\
\hline $\mathrm{CH}-\mathrm{CM}$ & .2193 & 0 & -- & .50 & 0 & .20 & 3.082 & 0.538 & 0.143 & 1.5 & 8.5 & 10.5 & 40.5 \\
\hline DH-IM & .1000 & .400 & .300 & .10 & 1.01 & .20 & 2.942 & 0.491 & 0.124 & 1.5 & 8.5 & 10.5 & 40.5 \\
\hline CH-IM & .2193 & 0 & -- & .10 & 1.01 & .20 & 3.030 & 0.510 & 0.130 & 1.5 & 8.5 & 10.5 & 40.5 \\
\hline
\end{tabular}

$564 \mathrm{CH}$ : Constant hazard (risk) model (equation 1).

565 IH: Increasing hazard (risk) model (equation 2).

566 DH: Decreasing hazard (risk) model (equation 6).

567 IF: Increasing fecundity model (equation 3).

568 CF: Constant fecundity model (equation 4).

569 DF: Declining fecundity model (equation 5).

570 FM: Fixed age of maturation at age $x_{2}$.

571 CM: Constant rate of maturation.

572 IM: Exponentially increasing rate of maturation. 
$573 \tau:$ age

$574 \quad l:$ length

$575 \mu:$ number of births per adult

$576 g$ : number of individuals reaching maturity per juvenile

577 


\section{Table 2}

579 Table 2. Nine ways of converting age-specific survival rates into a stage-specific survival rate. Note 580 lower-case $s$ is used for an age-specific survival rate and upper-case $S$ is used for a stage-specific 581 survival rate. $x_{i}$ is the first age class in the stage $i$, and $x_{j}$ is the first age class in stage $j=i+1$.

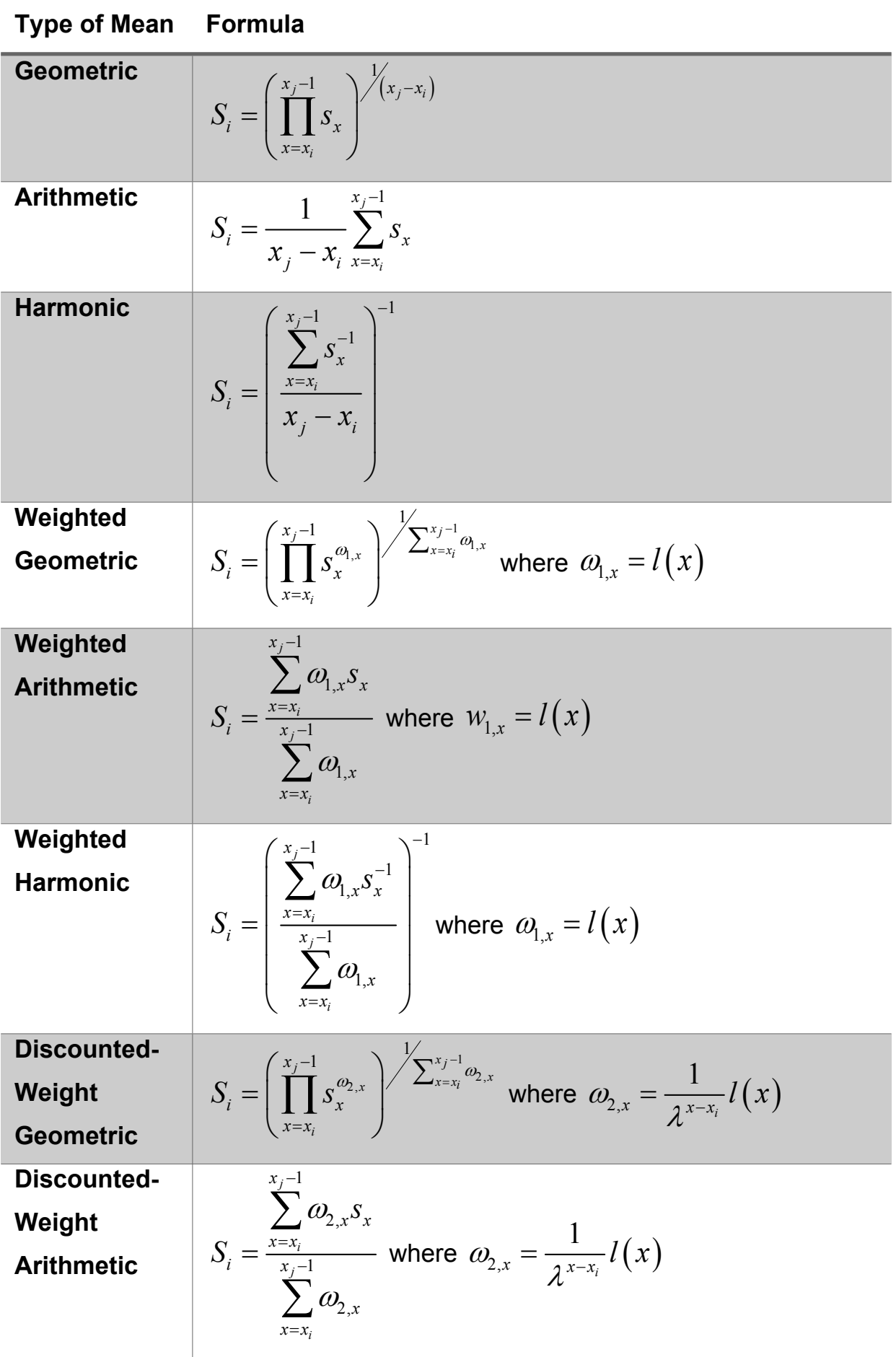




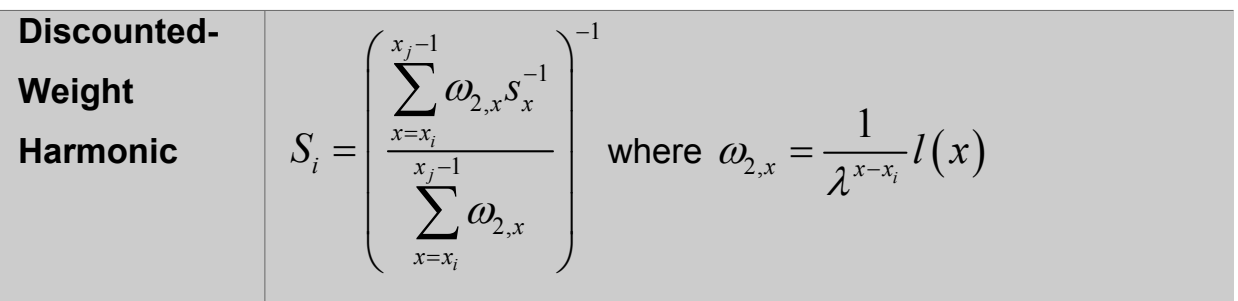

582

\section{Table 3}

584 Table 3. Three methods for the calculation of the conditional stage-transition rate $P_{j, i} . x_{i}$ is the first age 585 class in the stage $i$, and $x_{j}$ is the first age class in stage $j=i+1$.

MODEL Type of Transition Formula

\section{Rate}

\begin{tabular}{c|l|l}
\hline T1 & Matching Duration & $P_{j, i}=1-\frac{1}{x_{j}-x_{i}}$ \\
\hline T2 & $\begin{array}{l}\text { Matching proportion } \\
\text { transitioning }\end{array}$ & $P_{j, i}=\frac{l\left(x_{j}-1\right)}{\sum_{x=x_{i}}^{x_{j}-1} l(x)}$ \\
\hline T3 & $\begin{array}{l}\text { Matching proportion } \\
\text { transitioning with } \\
\text { discount }\end{array}$ & $P_{j, i}=\frac{\lambda^{-\left(x_{j}-x_{i}-1\right)} l\left(x_{j}-1\right)}{\sum_{x=x_{i}}^{x_{j}-1} \lambda^{-\left(x-x_{i}\right)} l(x)}$ \\
\hline
\end{tabular}

586

587

588 
589 Table 4

590 Table 4. Model types for estimating $\lambda$ and generation time

Models Description

Generation

Time

Calculation

\begin{tabular}{|c|c|c|}
\hline a & Euler-Lotka equation & $G_{1}$ or $G_{2}$ \\
\hline b & Age structured model with proportion mature & $G_{3}$ \\
\hline c & Age structured model with fixed age of maturity & $G_{3}$ \\
\hline d & Two-stage model with a geometric mean survival rate & $G_{3}$ \\
\hline e & Two-stage model with an arithmetic mean survival rate & $G_{3}$ \\
\hline $\mathbf{f}$ & Two-stage model with a harmonic mean survival rate & $G_{3}$ \\
\hline g & Two-stage model with a weighted geometric mean survival rate & $G_{3}$ \\
\hline $\mathbf{h}$ & Two-stage model with a weighted geometric mean survival rate & $G_{3}$ \\
\hline $\mathbf{i}$ & Two-stage model with a weighted harmonic mean survival rate & $G_{3}$ \\
\hline $\mathbf{j}$ & $\begin{array}{l}\text { Two-stage model with a weighted geometric mean survival rate } \\
\text { with } \lambda \text { used as discounting factor }\end{array}$ & $G_{3}$ \\
\hline $\mathbf{k}$ & $\begin{array}{l}\text { Two-stage model with a weighted geometric mean survival rate } \\
\text { with } \lambda \text { used as discounting factor }\end{array}$ & $G_{3}$ \\
\hline $\mathbf{l}$ & $\begin{array}{l}\text { Two-stage model with a weighted harmonic mean survival rate } \\
\text { with } \lambda \text { used as discounting factor }\end{array}$ & $G_{3}$ \\
\hline m & $\begin{array}{l}\text { Three-stage model with an arithmetic mean survival rate and } \\
\text { matching duration (T1) }\end{array}$ & $G_{3}$ \\
\hline $\mathbf{n}$ & $\begin{array}{l}\text { Three-stage model with an arithmetic mean survival rate and } \\
\text { matching proportion transitioning (T2) }\end{array}$ & $G_{3}$ \\
\hline o & $\begin{array}{l}\text { Three-stage model with an arithmetic mean survival rate and } \\
\text { matching proportion transitioning with discount (T3) }\end{array}$ & $G_{3}$ \\
\hline $\mathbf{p}$ & $\begin{array}{l}\text { Three-stage model with a weighted arithmetic mean survival rate } \\
\text { and matching duration (T1) }\end{array}$ & $G_{3}$ \\
\hline
\end{tabular}




\begin{tabular}{|l|l|l|}
\hline $\mathbf{q}$ & $\begin{array}{l}\text { Three-stage model with a weighted arithmetic mean survival rate } \\
\text { and matching proportion transitioning (T2) }\end{array}$ & $G_{3}$ \\
\hline $\mathbf{r}$ & $\begin{array}{l}\text { Three-stage model with a weighted arithmetic mean survival rate } \\
\text { and matching proportion transitioning with discount (T3) }\end{array}$ & $G_{3}$ \\
\hline $\mathbf{s}$ & $\begin{array}{l}\text { Three-stage model with a discounted-weight arithmetic mean } \\
\text { survival rate and matching duration (T1) }\end{array}$ & $G_{3}$ \\
\hline $\mathbf{t}$ & $\begin{array}{l}\text { Three-stage model with a discount-weight arithmetic mean } \\
\text { survival rate and matching proportion transitioning (T2) }\end{array}$ & $G_{3}$ \\
\hline $\mathbf{u}$ & $\begin{array}{l}\text { Three-stage model with a discount-weight arithmetic mean } \\
\text { survival rate and matching proportion transitioning with discount } \\
\text { (T3) }\end{array}$ & $G_{3}$ \\
\hline
\end{tabular}

591

592

\section{Table 5}

594 Table 5. Population growth rate estimated using the iterative method. Six different life history strategies 595 of organisms with delayed maturation at three different levels of true $\lambda$ were investigated.

Life History Types (see Table 1)

\begin{tabular}{c|r|r|r|r|r|r|}
\hline True $\lambda$ & D.H - F.M. & C.H. - F.M. & D.H. - C.M. & C.H. - C.M. & D.H. - I.M. & C.H. - I.M. \\
\cline { 2 - 7 }$\lambda=0.90$ & 0.900 & 0.903 & 0.899 & 0.902 & 0.900 & 0.902 \\
$\lambda=1.00$ & 0.994 & 1.000 & 0.992 & 0.995 & 0.994 & 0.999 \\
$\lambda=1.10$ & 1.090 & 1.100 & 1.085 & 1.089 & 1.090 & 1.096
\end{tabular}




\section{Figure Legend}

599 Figure 1. Survivorship, fecundity, and maturation schedule. (A) Survivorship of individuals under a

600

601

602

603

604

605

606

607

608

609

610

611

612

613

614

615

616

617

618

619

620

621

622

623

624

625

626

627

628 constant risk (solid line, equation1) and an exponentially increasing risk (dotted line, equation 2). (B) Fecundity as a function of age: increasing fecundity (solid line; equation 3), constant fecundity (dotted line; equation 4), and exponentially declining fecundity (dash-dot; equation 5). (C) Survivorship of individuals under a declining risk (solid line; equation 6) and a constant risk (dashed line; equation 1). (D) Proportion of individuals mature with the increasing rate of maturation (solid line), the fixed age of maturation (dotted line), and the constant rate of maturation (dash dot).

Figure 2 . Finite asymptotic population growth rate $\lambda$ when the true $\lambda$ is 1.000 for early maturation types. Each panel represents different life history type as was defined in Table 1. Each bar represents a different model for estimating $\lambda$. See Table 4 for model types. (Black, Euler-Lotka equation; Gray, age-structured model; Magenta, stage-structured with ordinary mean; Cyan, stage-structured with weighted mean; Yellow, stage-structured with weight mean with discount)

Figure 3. Finite asymptotic population growth rate $\lambda$ when the true $\lambda$ is 0.900 for early maturation types. Each panel represents different life history type as was defined in Table 1. Each bar represents a different model for estimating $\lambda$. See Table 4 for model types. (Black, Euler-Lotka equation; Gray, age-structured model; Magenta, stage-structured with ordinary mean; Cyan, stage-structured with weighted mean; Yellow, stage-structured with weight mean with discount)

Figure 4. Finite asymptotic population growth rate $\lambda$ when the true $\lambda$ is 1.100 for early maturation types. Each panel represents different life history type as was defined in Table 1. Each bar represents a different model for estimating $\lambda$. See Table 4 for model types. (Black, Euler-Lotka equation; Gray, age-structured model; Magenta, stage-structured with ordinary mean; Cyan, stage-structured with weighted mean; Yellow, stage-structured with weight mean with discount)

Figure 5. Finite asymptotic population growth rate $\lambda$ when the true $\lambda$ is 1.000 for delayed maturation types. Each panel represents different life history type as was defined in Table 1. Each bar represents a different model for estimating $\lambda$. See Table 4 for model types. (Black, Euler-Lotka equation; Gray, age-structured; Blue, stage-structured with an arithmetic mean; Green, stage-structured with a weighted arithmetic mean; Red, stage-structured with a discounted-weight arithmetic mean)

Figure 6. Finite asymptotic population growth rate $\lambda$ when the true $\lambda$ is 0.900 for delayed maturation types. Each panel represents different life history type as was defined in Table 1. Each bar represents a 
629

630

631

632

633

634

635

636

637

638

639

640

641

642

643

644

645

646

647

648

649

650

651

652

653

654

655

656

657

658

different model for estimating $\lambda$. See Table 4 for model types. (Black, Euler-Lotka equation; Gray, age-structured; Blue, stage-structured with an arithmetic mean; Green, stage-structured with a weighted arithmetic mean; Red, stage-structured with a discounted-weight arithmetic mean)

Figure 7. Finite asymptotic population growth rate $\lambda$ when the true $\lambda$ is 1.100 for delayed maturation types. Each panel represents different life history type as was defined in Table 1. Each bar represents a different model for estimating $\lambda$. See Table 4 for model types. (Black, Euler-Lotka equation; Gray, age-structured; Blue, stage-structured with an arithmetic mean; Green, stage-structured with a weighted arithmetic mean; Red, stage-structured with a discounted-weight arithmetic mean)

Figure 8. Generation time for the early maturation types where the true finite population growth rate $\lambda$ is 1.000 for delayed maturation types. Each panel represents different life history type as was defined in Table 1. Each bar represents a different model for estimating $\lambda$ and generation time. See Table 4 for model types. (Black, Euler-Lotka equation; Gray, age-structured model; Magenta, stage-structured with ordinary mean; Cyan, stage-structured with weighted mean; Yellow, stagestructured with weight mean with discount)

Figure 9. Generation time for the early maturation types where the true finite population growth rate $\lambda$ is 0.900. Each panel represents different life history type as was defined in Table 1. Each bar represents a different model for estimating $\lambda$ and generation time. See Table 4 for model types. (Black, Euler-Lotka equation; Gray, age-structured model; Magenta, stage-structured with ordinary mean; Cyan, stage-structured with weighted mean; Yellow, stage-structured with weight mean with discount)

Figure 10. Generation time for the early maturation types where the true finite population growth rate $\lambda$ is 1.100. Each panel represents different life history type as was defined in Table 1. Each bar represents a different model for estimating $\lambda$ and generation time. See Table 4 for model types. (Black, Euler-Lotka equation; Gray, age-structured model; Magenta, stage-structured with ordinary mean; Cyan, stage-structured with weighted mean; Yellow, stage-structured with weight mean with discount)

Figure 11. Generation time for the delayed maturation types where the true finite population growth rate $\lambda$ is 1.000. Each panel represents different life history type as was defined in Table 1. Each bar represents a different model for estimating $\lambda$ and generation time. See Table 4 for model types. (Black, Euler-Lotka equation; Gray, age-structured; Blue, stage-structured with an arithmetic 
659

660

661

662

663

664

665

666

667

668

669

670

671

672

673 mean; Green, stage-structured with a weighted arithmetic mean; Red, stage-structured with a discounted-weight arithmetic mean)

Figure 12. Generation time for the delayed maturation types where the true finite population growth rate $\lambda$ is 0.900. Each panel represents different life history type as was defined in Table 1. Each bar represents a different model for estimating $\lambda$ and generation time. See Table 4 for model types. (Black, Euler-Lotka equation; Gray, age-structured; Blue, stage-structured with an arithmetic mean; Green, stage-structured with a weighted arithmetic mean; Red, stage-structured with a discounted-weight arithmetic mean)

Figure 13. Generation time for the delayed maturation types where the true finite population growth rate $\lambda$ is 1.100. Each panel represents different life history type as was defined in Table 1. Each bar represents a different model for estimating $\lambda$ and generation time. See Table 4 for model types. (Black, Euler-Lotka equation; Gray, age-structured; Blue, stage-structured with an arithmetic mean; Green, stage-structured with a weighted arithmetic mean; Red, stage-structured with a discounted-weight arithmetic mean) 


\section{Figure 1}
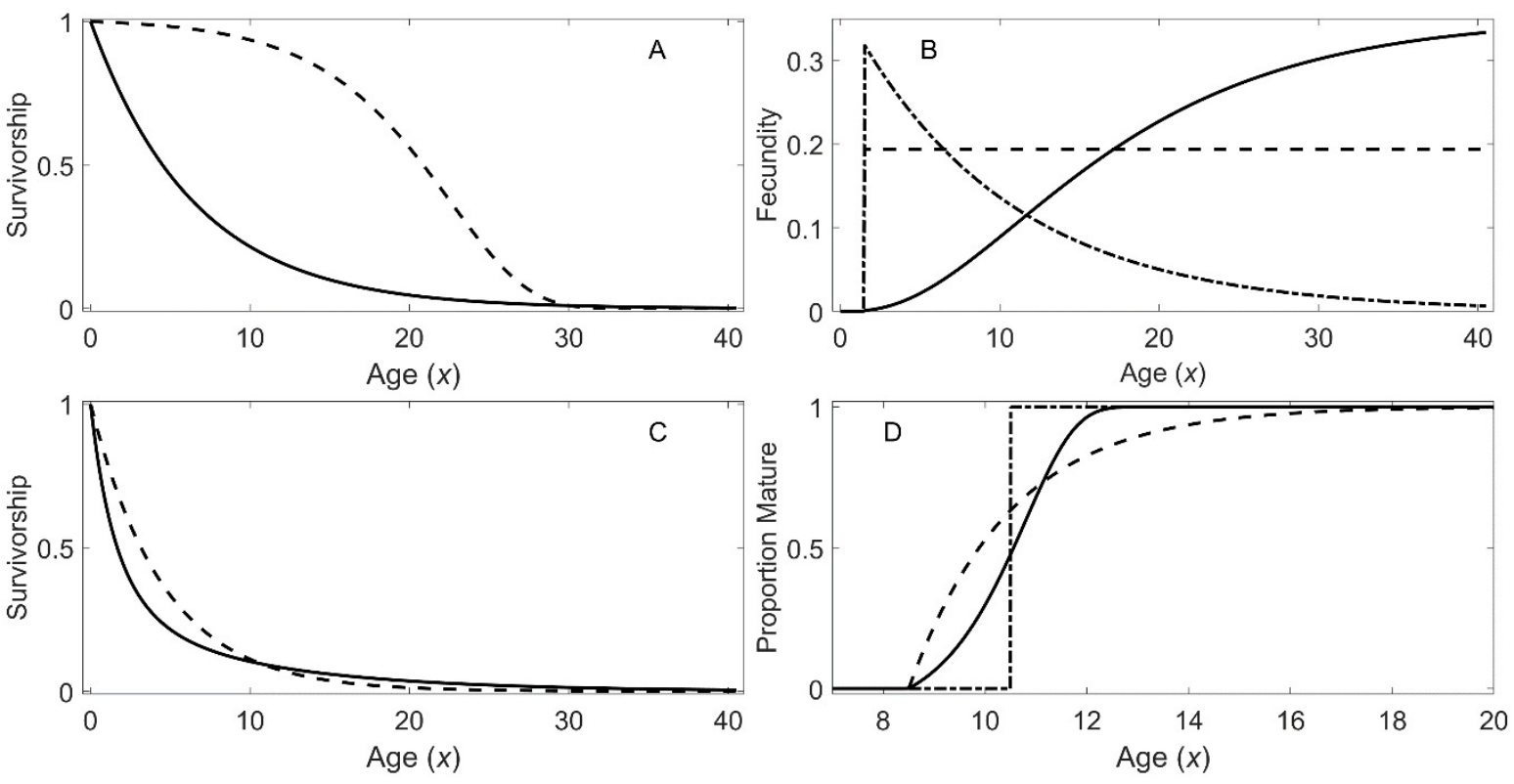

675

676

677 


\section{Figure 2}

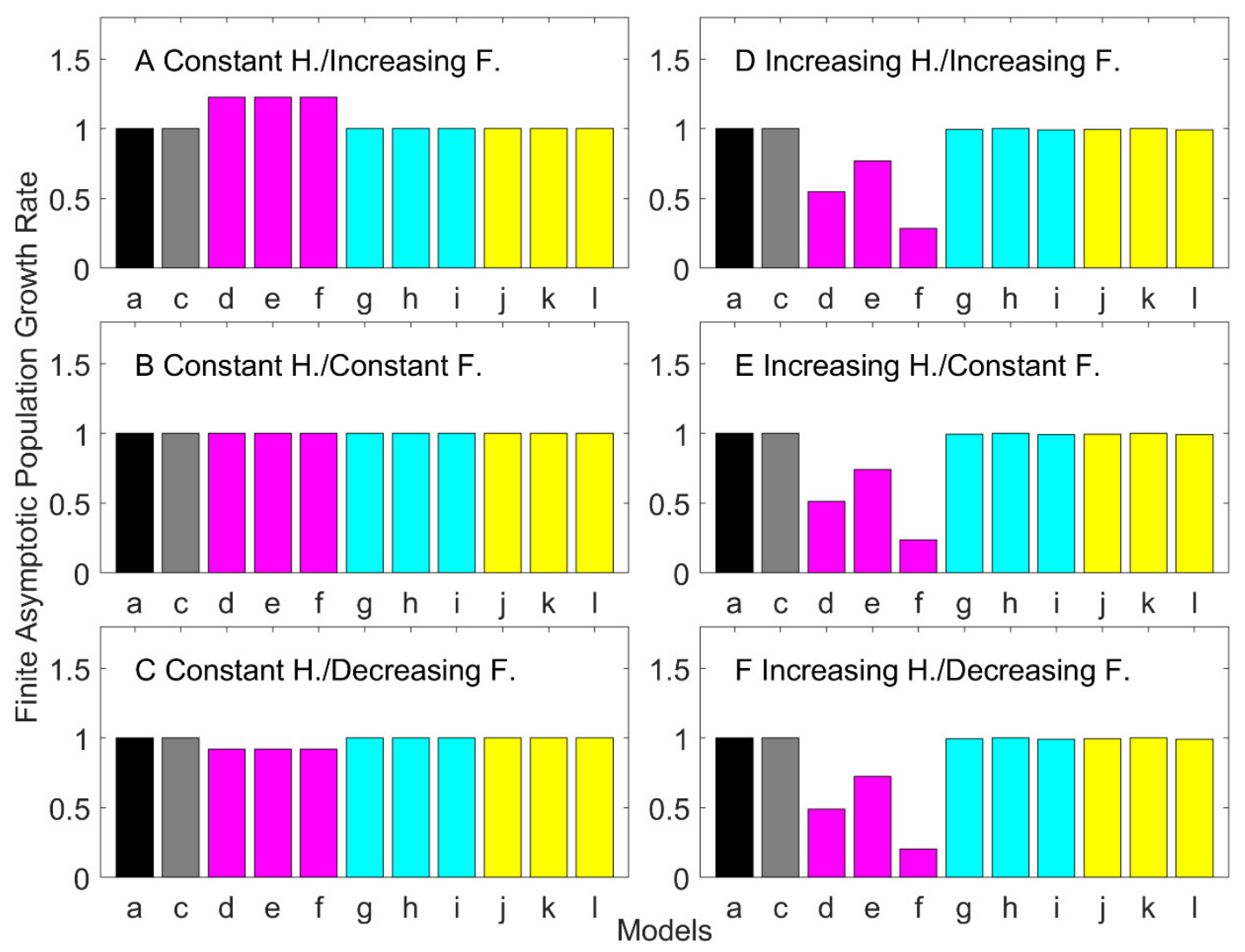

679

680

681 


\section{Figure 3}

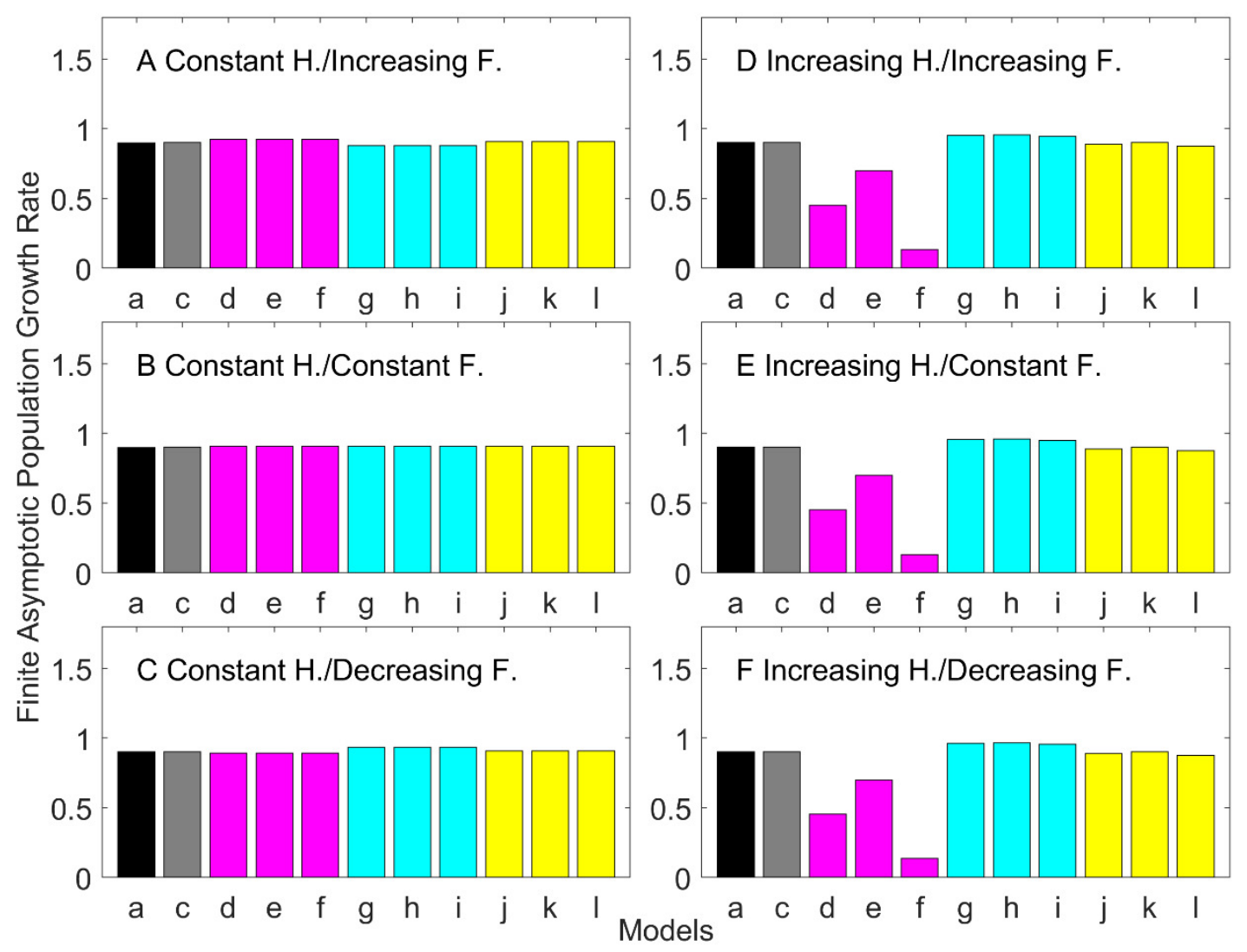

683

684 


\section{Figure 4}

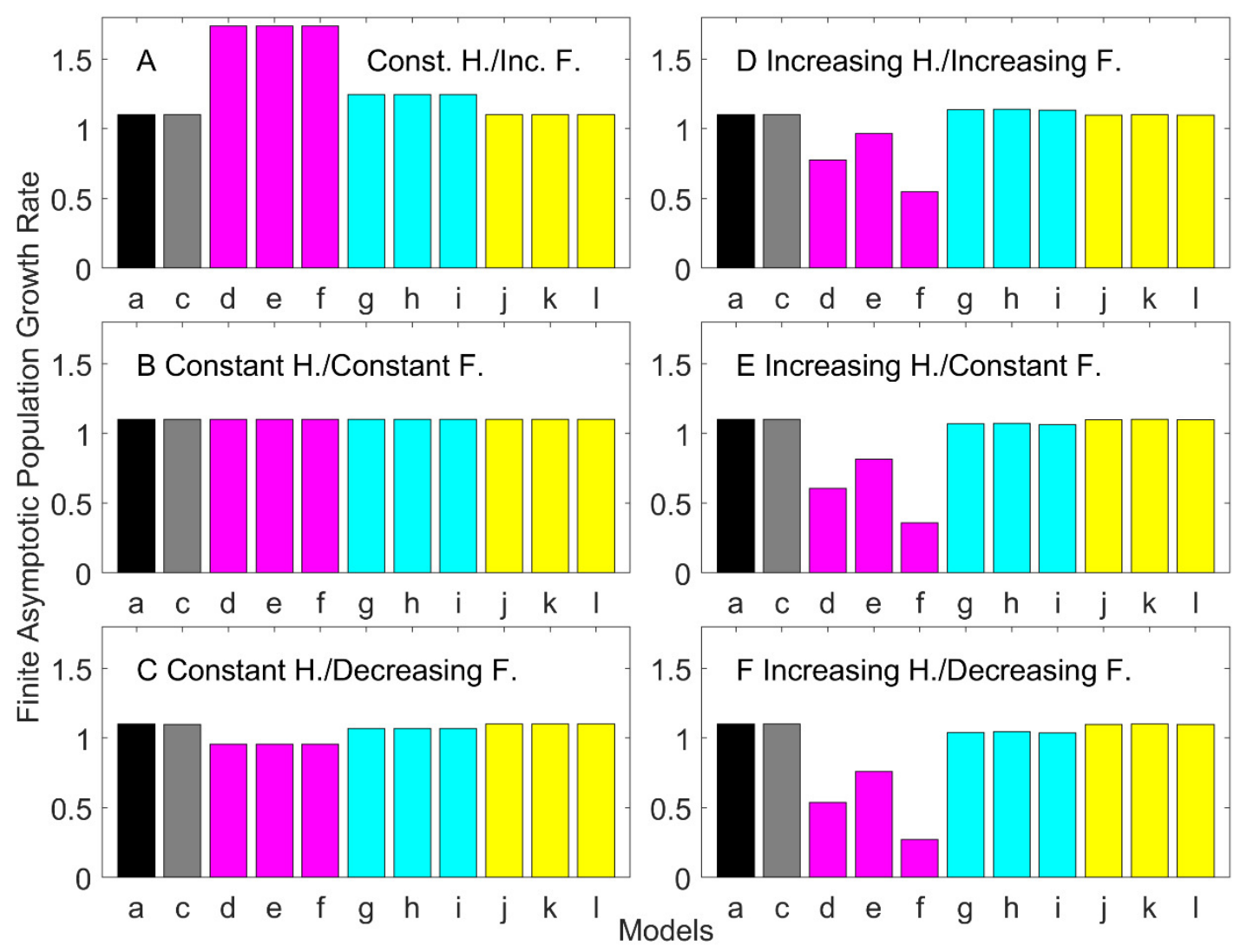

686

687 


\section{Figure 5}

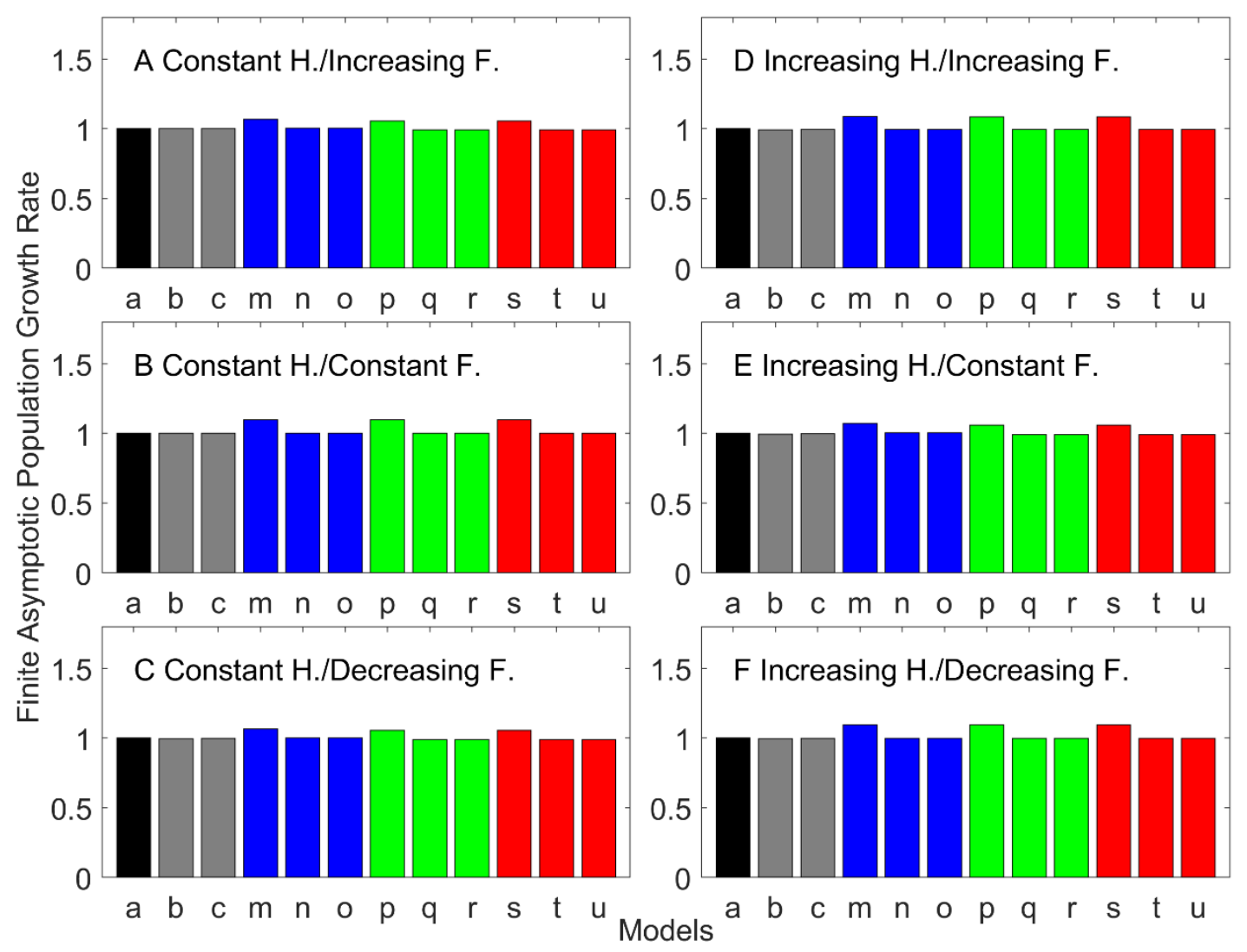

689

690

691 
692 Figure 6

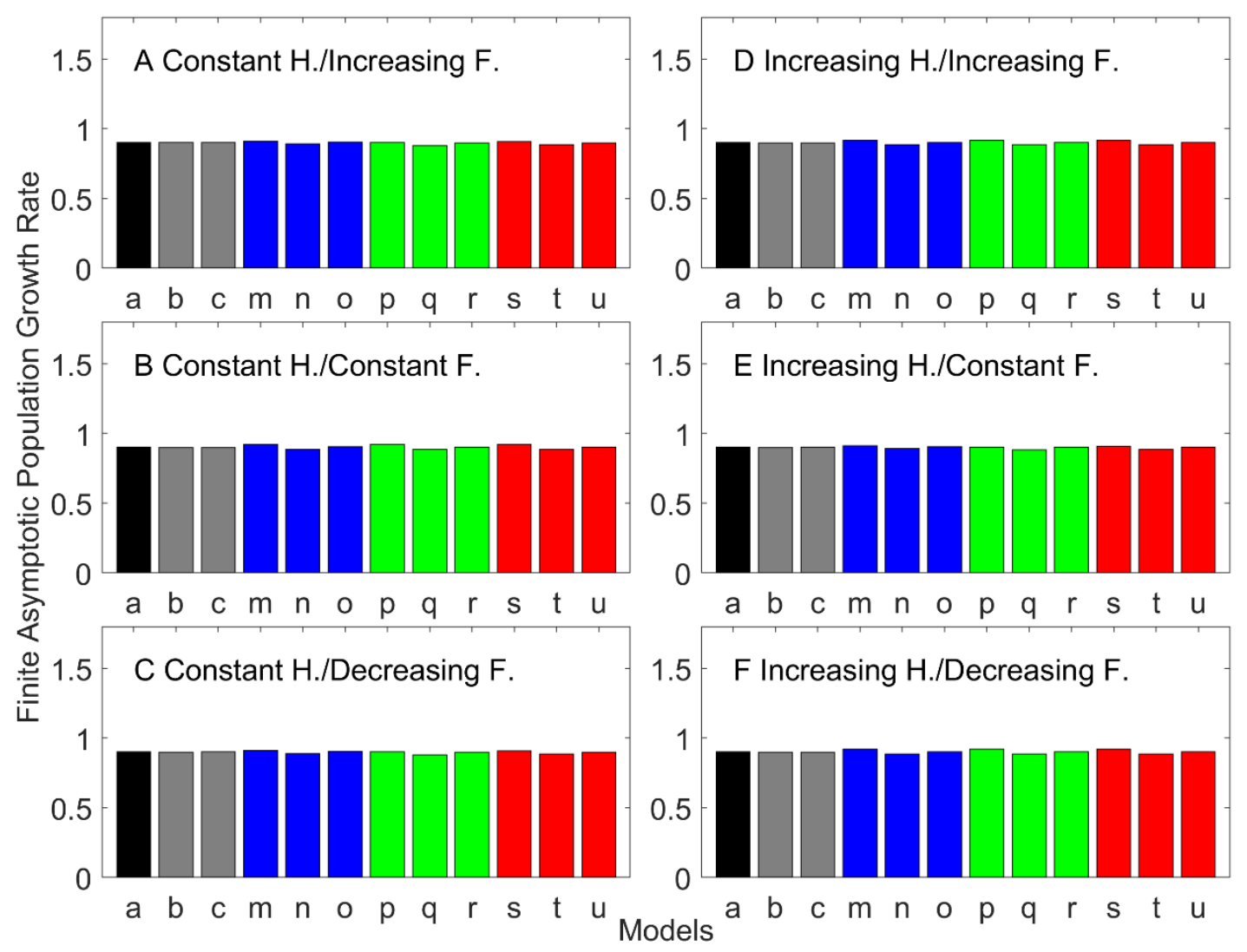

693

694

695 


\section{Figure 7}

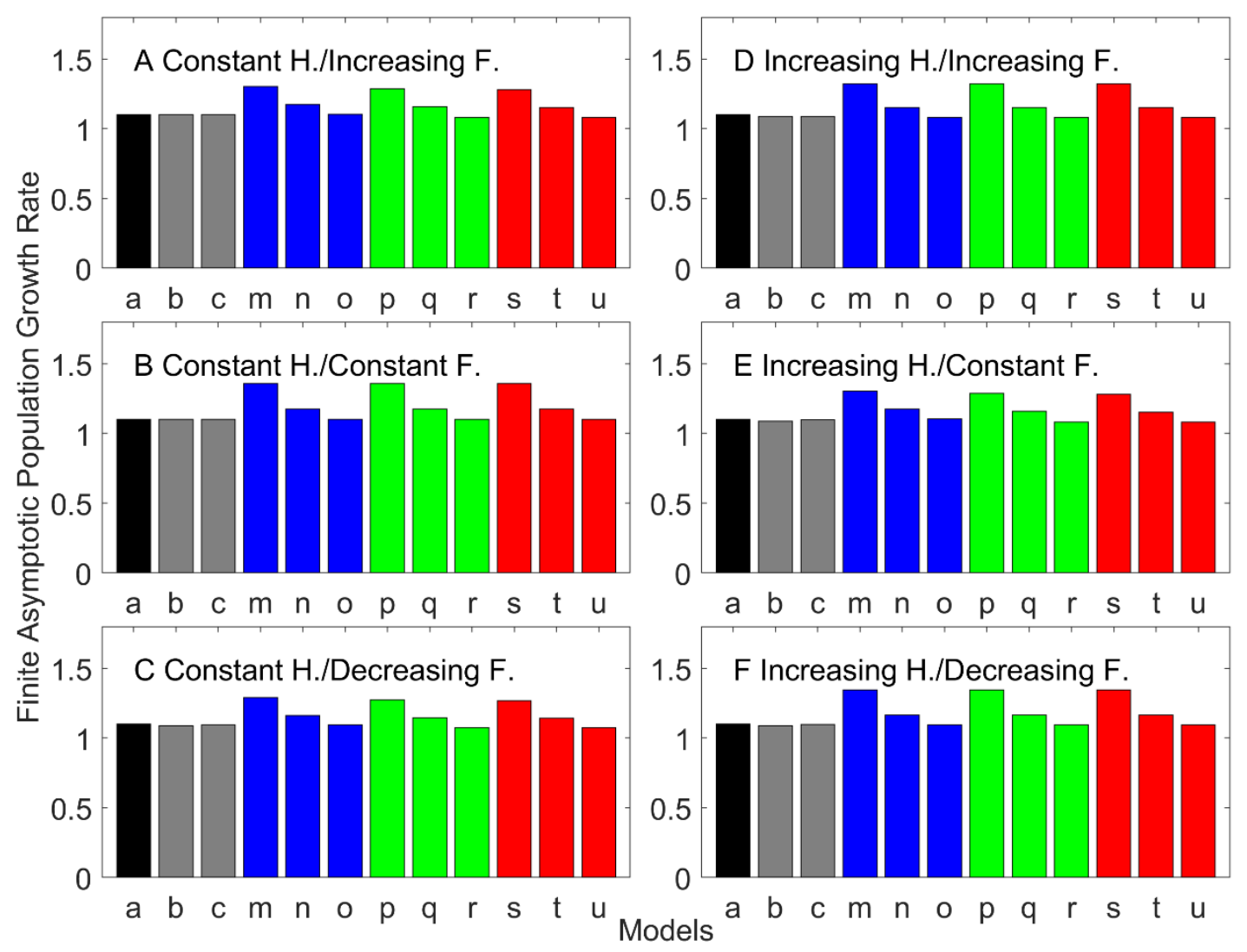

697

698 


\section{Figure 8}

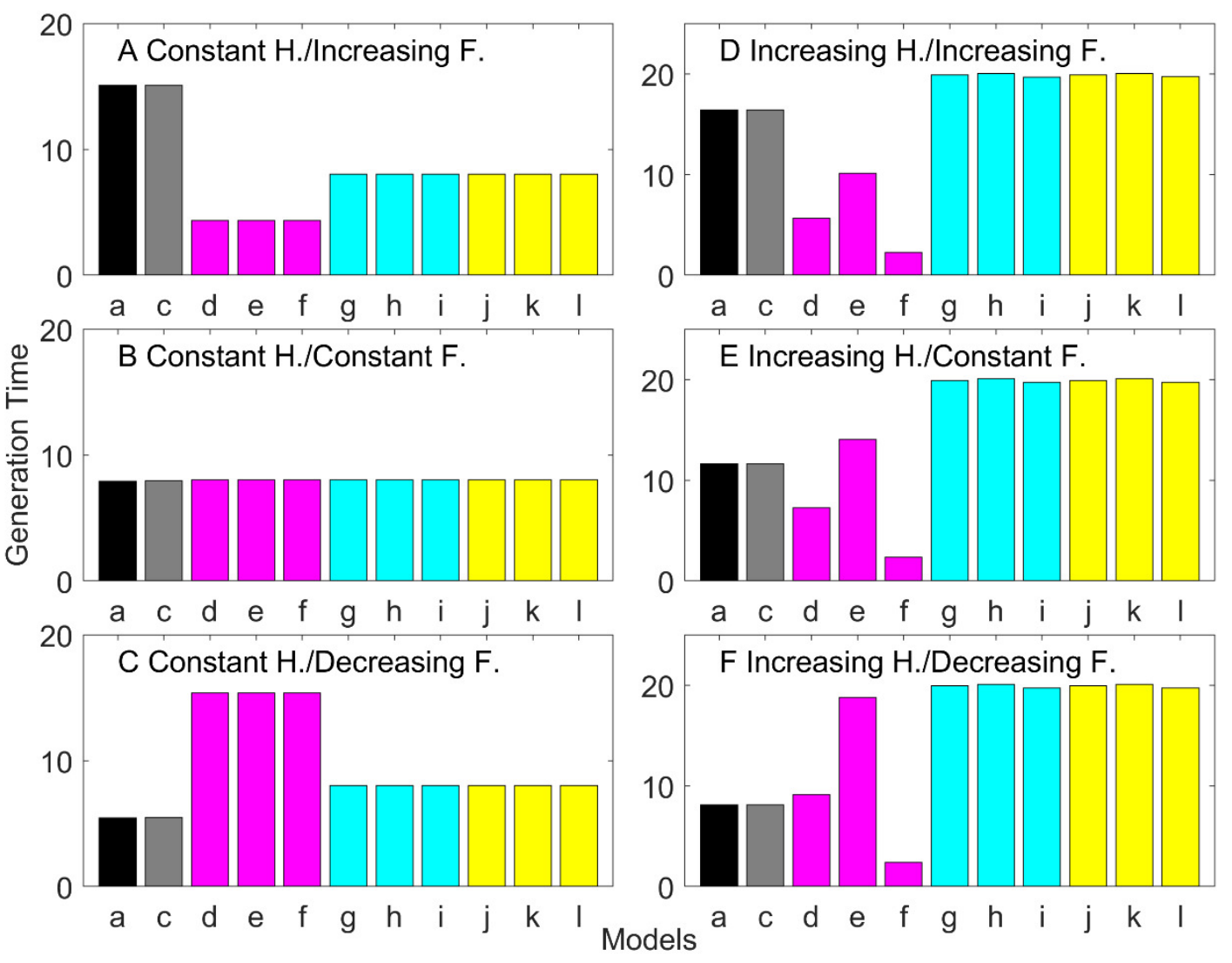

700

701

702 


\section{Figure 9}
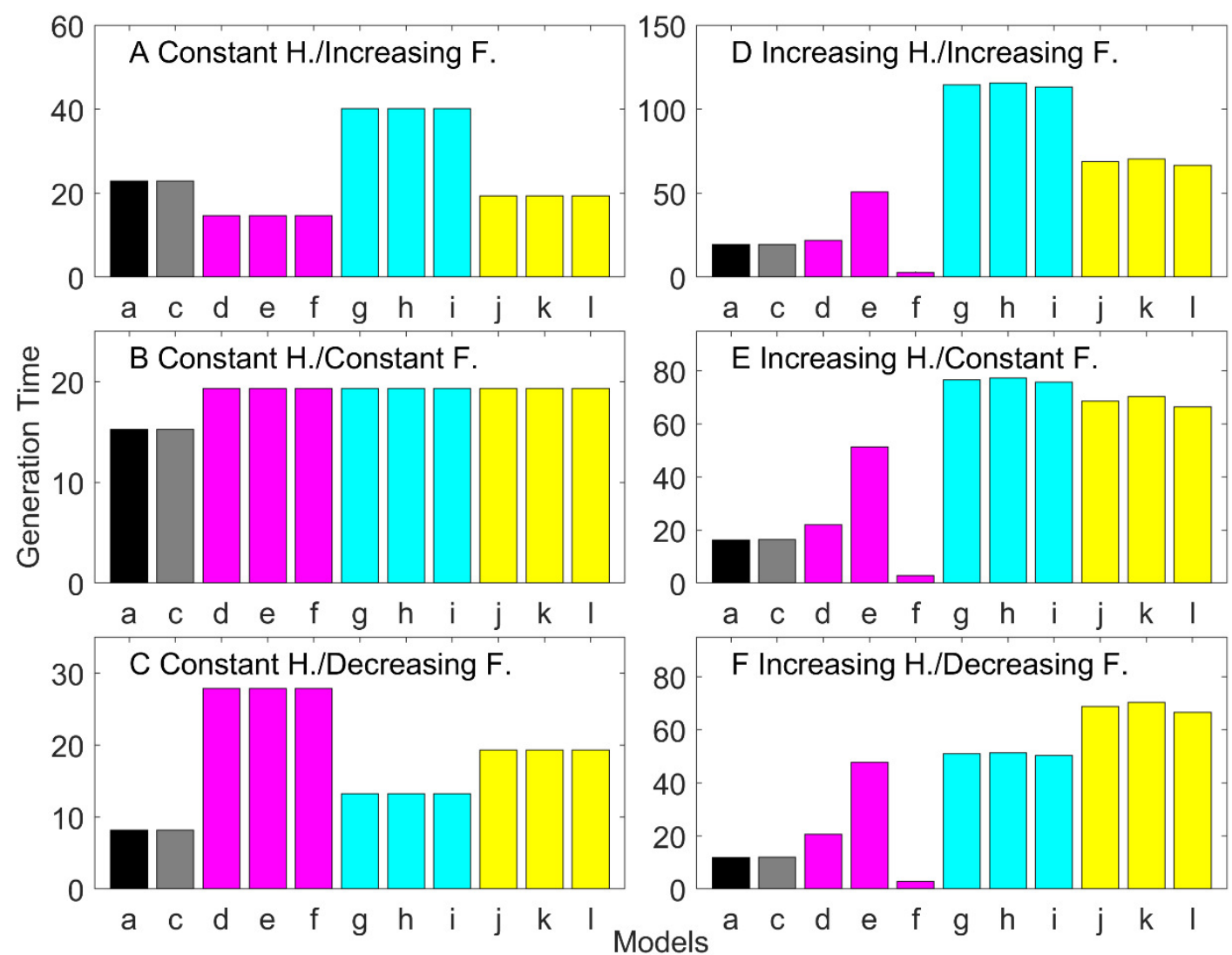

704

705 


\section{Figure 10}

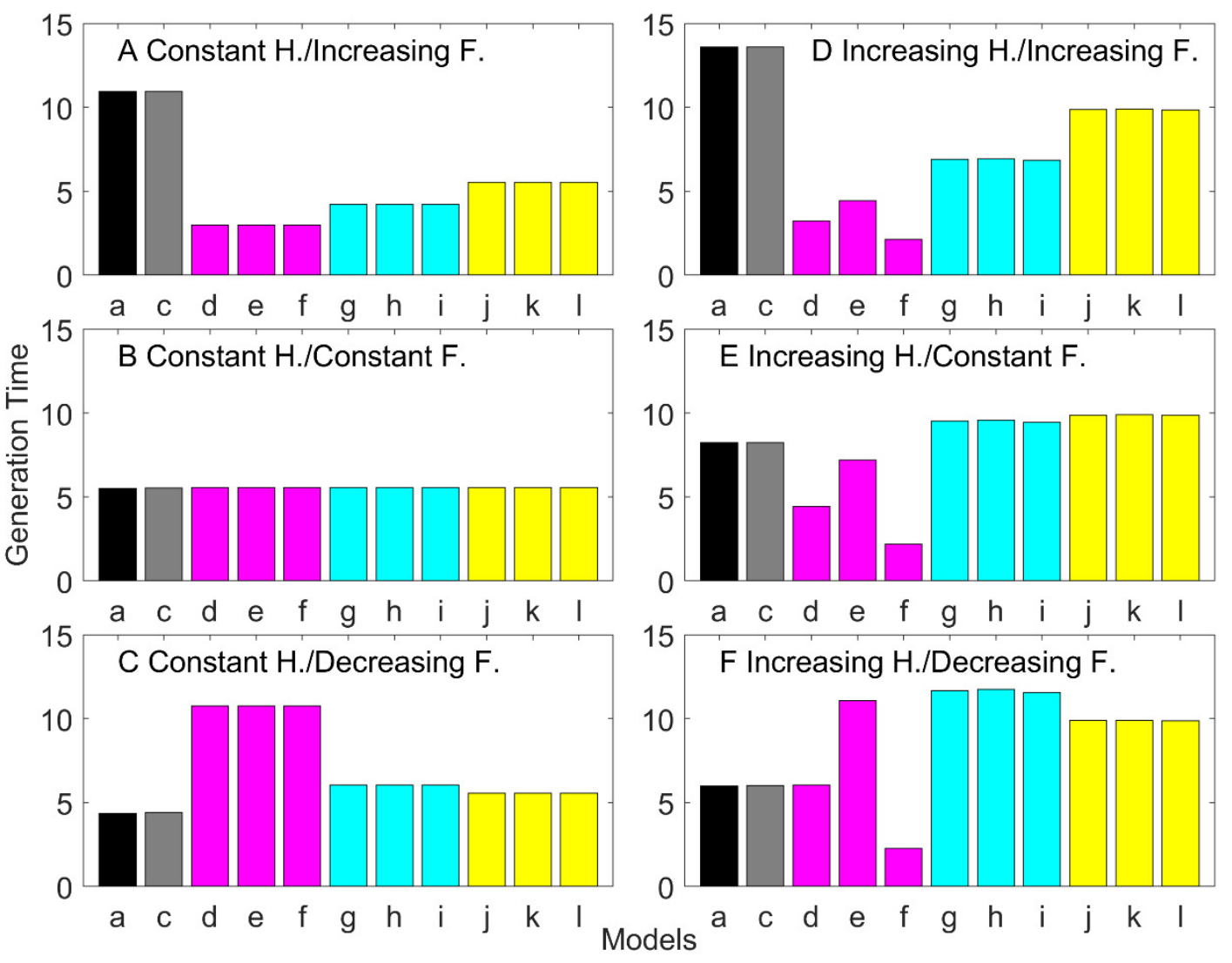

707

708 


\section{Figure 11}

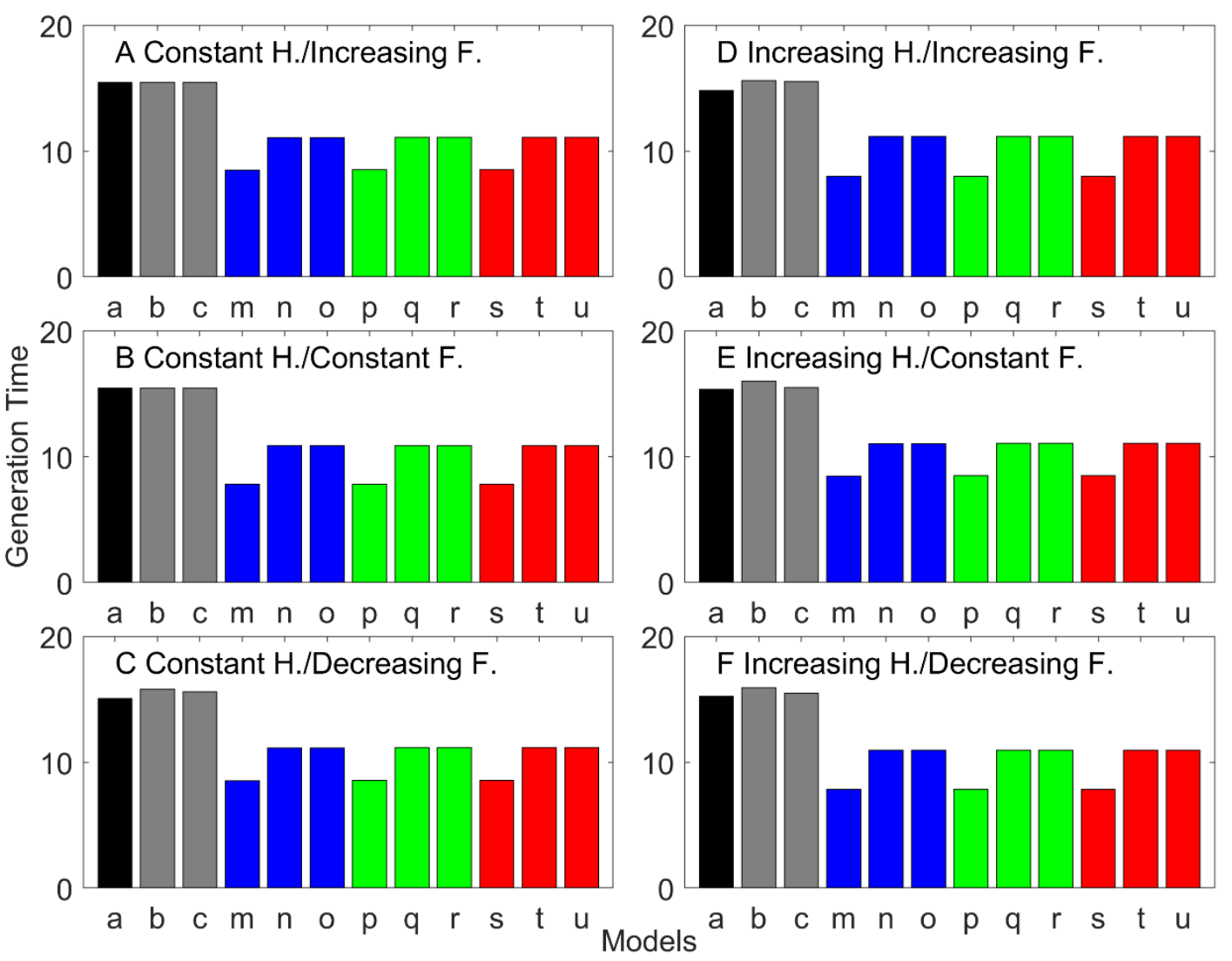

710

711 


\section{Figure 12}

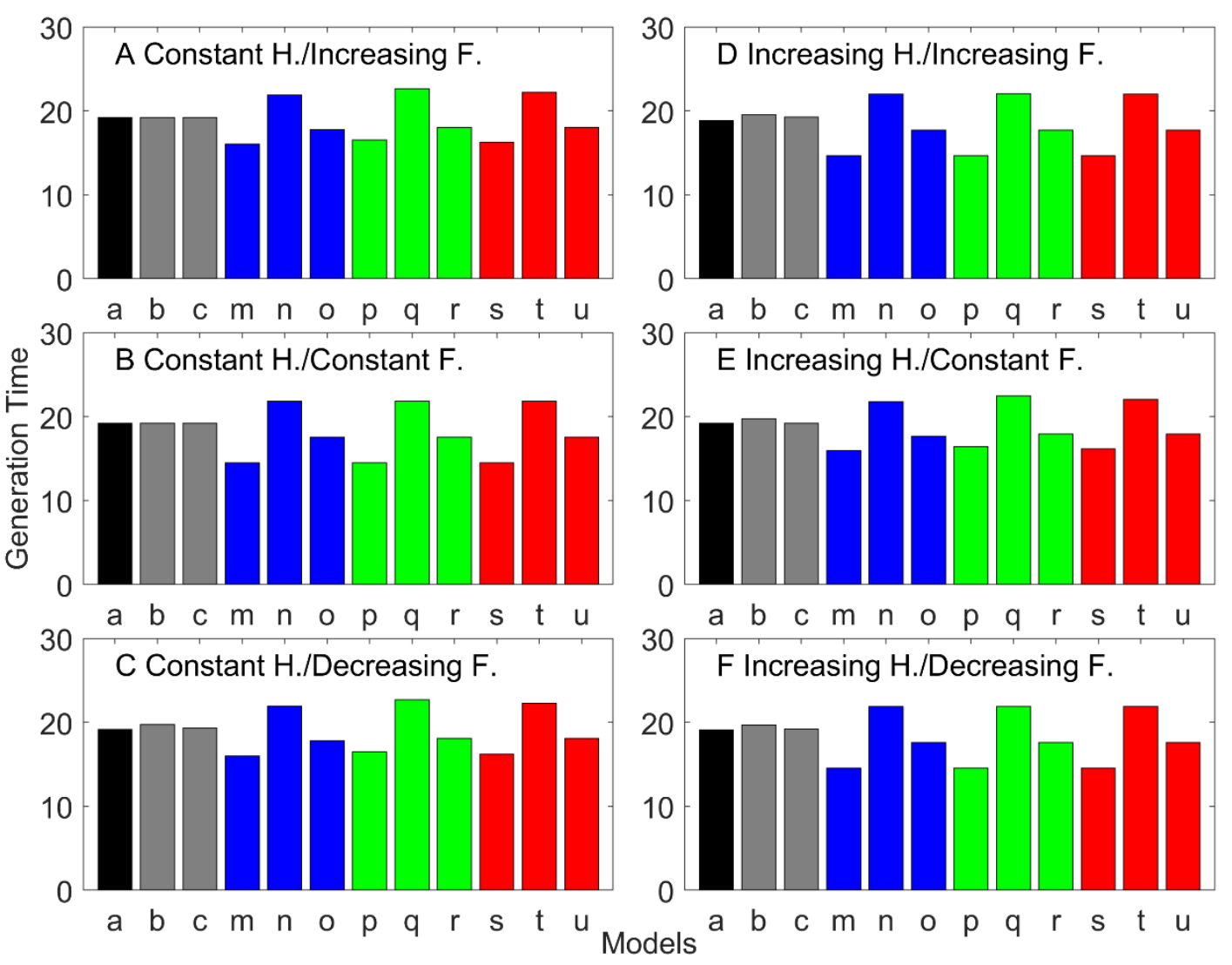

713

714

715 


\section{Figure 13}
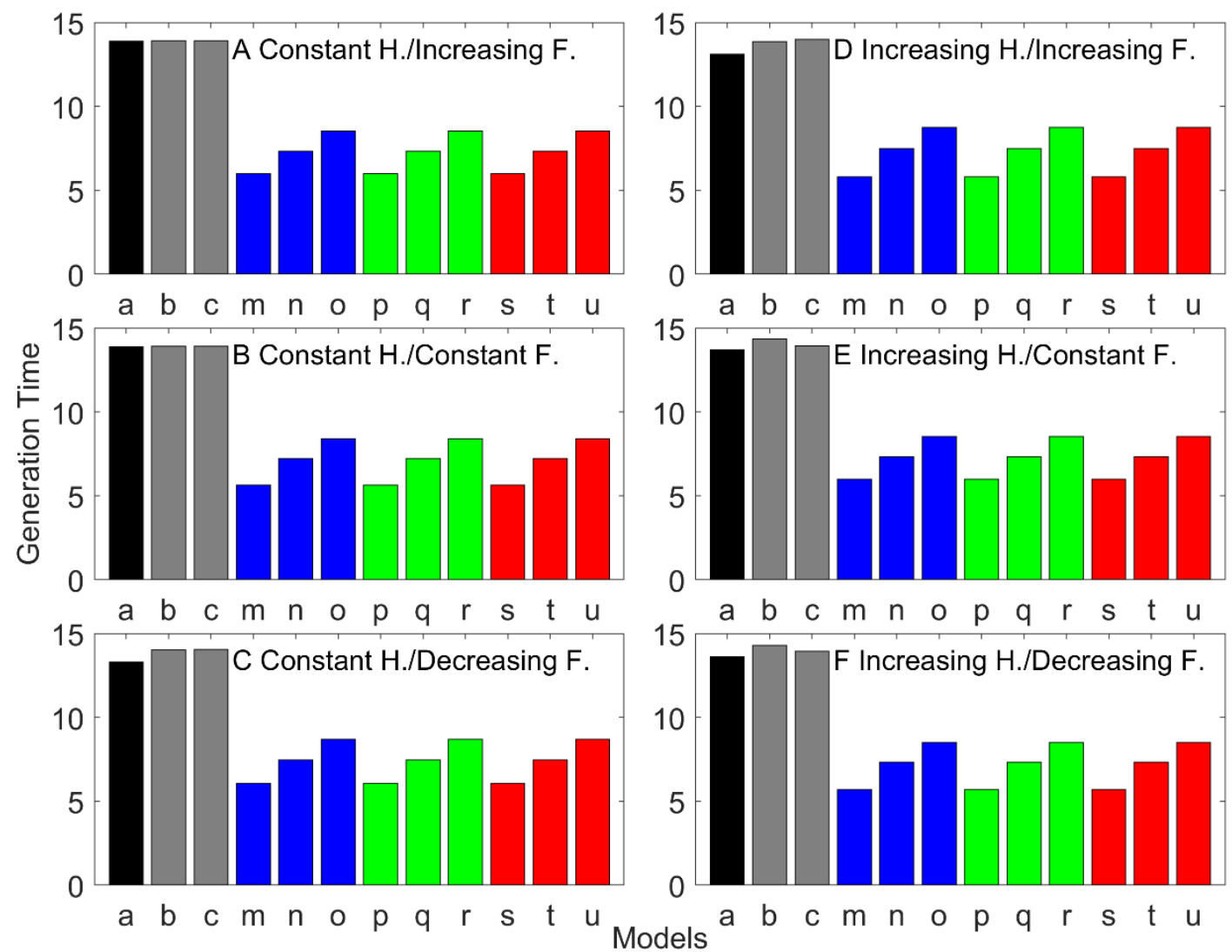

717

718 